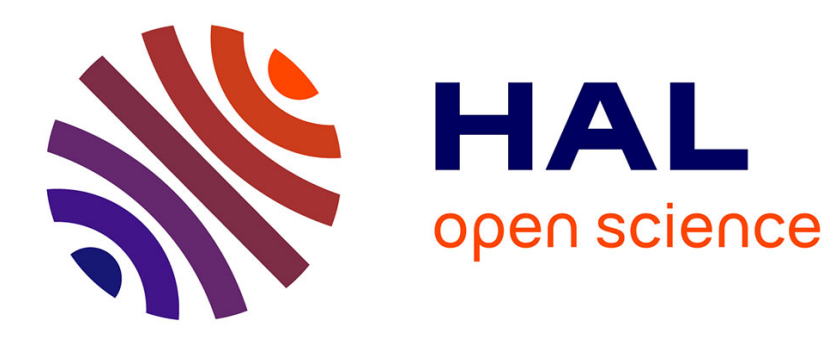

\title{
On Hom-Lie superbialgebras
}

\author{
Mohamed Fadous, Sami Mabrouk, Abdenacer Makhlouf
}

\section{To cite this version:}

Mohamed Fadous, Sami Mabrouk, Abdenacer Makhlouf. On Hom-Lie superbialgebras. Communications in Algebra, 2018, 47 (1), pp.114-137. 10.1080/00927872.2018.1468900 . hal-03498260

\section{HAL Id: hal-03498260 https://hal.science/hal-03498260}

Submitted on 20 Dec 2021

HAL is a multi-disciplinary open access archive for the deposit and dissemination of scientific research documents, whether they are published or not. The documents may come from teaching and research institutions in France or abroad, or from public or private research centers.
L'archive ouverte pluridisciplinaire HAL, est destinée au dépôt et à la diffusion de documents scientifiques de niveau recherche, publiés ou non, émanant des établissements d'enseignement et de recherche français ou étrangers, des laboratoires publics ou privés. 


\title{
On Hom-Lie superbialgebras
}

\author{
Mohamed Fadous, Sami Mabrouk, Abdenacer Makhlouf
}

\begin{abstract}
The purpose of this paper is to generalize to $\mathbb{Z}_{2}$-graded case the study of Hom-Lie bialgebras which were discussed first by D. Yau, then by C. Bai and Y. Sheng. We provide different ways for constructing Hom-Lie superbialgebras. Also we define Matched pairs, Manin supertriples and discuss their relationships. Moreover, we study coboundary and triangular Hom-Lie bialgebras, as well as infinitesimal deformations of the cobracket.
\end{abstract}

Keywords :Hom-Lie superalgebra, coboundary Hom-Lie bialgebra, triangular Hom-Lie bialgebra 2010 Mathematics Subject Classification : 17B62, $17 \mathrm{~B} 37$.

\section{Introduction}

Lie bialgebras were introduced by V. Drinfel'd in 17, 8, they are infinitesimal versions of compatible Poisson structures on Lie groups and maybe viewed as the Lie-theoretic case of a bialgebra. He raised various problems related to quantum groups and quantization. The study of quasi-triangular quantum groups involves the solutions of the quantum Yang-Baxter equations. In the classical limit, the solutions of the classical Yang-Baxter equations provide examples of Lie bialgebras. Since then a huge research activity was dedicated to these kind of algebraic structures.

The aim of this paper is to define and study Hom-Lie superbialgebras which are Hom-type generalization of Lie superbialgebras. Hom-Lie superbialgebras are Hom-Lie superalgebras provided with a cobracket and a compatibility condition. Motivated by examples of $q$-deformations of algebras of vector fields, J. Hartwig, D. Larsson, and S. Silvestrov introduced the notion of Hom-Lie algebra in [12, as a generalization of Lie algebras where the Jacobi condition is twisted by a Homomorphism. The graded case of Hom-Lie algebras were studied first by F. Ammar and the last author in 1, while Hom-Lie bialgebras were discussed by D. Yau, then by C. Bai and Y. Sheng. Recently L. Cai and Y. Sheng presented a slightly different approach of Hom-Lie bialgebras called purely Hom-Lie bialgebras in [5].

The paper is organized as follows, in the first section we provide the relevant definitions and some properties about Hom-Lie superbialgebras. Moreover, we give some key constructions and a classification of 3-dimensional Hom-Lie superbialgebras with 2-dimensional even part. In Section 2, We define Matched pairs and Manin supertriples, then we establish their relationships with Hom-Lie superbialgebras. We construct a Hom-Lie superalgebra structure on the direct sum of two Hom-Lie superalgebras $(\mathfrak{g},[\cdot, \cdot], \phi)$ and $\left(\mathfrak{g}^{\prime},[\cdot, \cdot]^{\prime}, \phi^{\prime}\right)$, such that $\mathfrak{g}$ is a $\mathfrak{g}^{\prime}$-module and $\mathfrak{g}^{\prime}$ is a $\mathfrak{g}$-module, also we construct a Hom-Lie superbialgebra structure on the direct sum $\mathfrak{g} \oplus \mathfrak{g}^{*}$ where $\mathfrak{g}^{*}$ is the dual superspace of $\mathfrak{g}$. Section 3 is dedicated to coboundary Hom-Lie superbialgebras and quasi-triangular Hom-Lie superbialgebras. We show how a coboundary or quasi-triangular Hom-Lie superbialgebra can be constructed from a Hom-Lie superalgebra and an $r$-matrix. In the last section, we study perturbation of cobrackets in Hom-Lie superbialgebras, following Drinfel'd's perturbation theory of quasi-Hopf algebras. We describe Hom-Lie superbialgebras obtained by infinitesimal deformations of the cobracket.

\section{Basics and Classification of Hom-Lie superbialgebras}

In this section, we introduce and study Hom-Lie superbialgebras, which are Hom-type version of Lie superbialgebras, see 7, 8. We extend to graded case the definition of Hom-Lie bialgebra introduced in [27. We show that the dual of a finite dimensional Hom-Lie superbialgebra is also a Hom-Lie superbialgebra (Theorem 1.18), generalizing the self-dual property of Hom-Lie bialgebras. 
First, let us start by fixing some definitions and notations. Let $\mathfrak{L}=\mathfrak{L}_{\overline{0}} \oplus \mathfrak{L}_{\overline{1}}$ be a $\mathbb{Z}_{2}$-graded vector space over an arbitrary field $\mathbb{K}$ of characteristic 0 . In the sequel, we will consider only element which are $\mathbb{Z}_{2}$-homogeneous. For $x \in \mathfrak{L}$, we denote by $|x| \in \mathbb{Z}_{2}$ its parity, i.e., $x \in \mathfrak{L}_{|x|}$. We denote by $\tau$ the super-twist map of $\mathfrak{L} \otimes \mathfrak{L}$ namely, $\tau(x \otimes y)=(-1)^{|x||y|} y \otimes x$ for $x, y \in \mathfrak{L}$. The super-cyclic map $\xi$ permutes the coordinates of $\mathfrak{L} \otimes \mathfrak{L} \otimes \mathfrak{L}$, it is defined as

$$
\xi=(\mathbf{1} \otimes \tau) \cdot(\tau \otimes \mathbf{1}): x \otimes y \otimes z \mapsto(-1)^{|x|(|y|+|z|)} y \otimes z \otimes x,
$$

for $x, y, z \in \mathfrak{L}$, where 1 is the identity map on $\mathfrak{L}$. We denote by $\mathfrak{L}^{*}=\operatorname{Hom}(\mathfrak{L}, \mathbb{K})$ the linear dual of $\mathfrak{L}$. For $\phi \in \mathfrak{L}^{*}$ and $x \in \mathfrak{L}$, we often use the adjoint notation $\langle\phi, x\rangle$ for $\phi(x) \in \mathbb{K}$.

For a linear map $\Delta: \mathfrak{L} \rightarrow \mathfrak{L} \otimes \mathfrak{L}$ (comultiplication), we use Sweedler's notation $\Delta(x)=\sum_{(x)} x_{1} \otimes x_{2}$ for $x \in \mathfrak{L}$. We will often omit the summation $\operatorname{sign} \sum_{(x)}$ to make it simple. The parity $|r|$ of $r \in \mathfrak{L}^{\otimes 2}$ is defined as follows : since we assume $r$ homogenous, there exists $|r| \in \mathbb{Z}_{2}$, such that $r$ can be written as $r=\sum r_{1} \otimes r_{2} \in \mathfrak{L}^{\otimes 2}, r_{1}, r_{2}$ are homogenous elements with $|r|=\left|r_{1}\right|+\left|r_{2}\right|$.

Definition 1.1. ([1]). A Hom-Lie superalgebra is a triple $(\mathfrak{L},[\cdot, \cdot], \alpha)$ consisting of a superspace $\mathfrak{L}$, an even bilinear map $[\cdot, \cdot]: \mathfrak{L} \times \mathfrak{L} \rightarrow \mathfrak{L}$ and an even superspace homomorphism $\alpha: \mathfrak{L} \rightarrow \mathfrak{L}$ satisfying

$$
\begin{gathered}
{[x, y]=-(-1)^{|x||y|}[y, x]} \\
(-1)^{|x||z|}[\alpha(x),[y, z]]+(-1)^{|z||y|}[\alpha(z),[x, y]]+(-1)^{|y||x|}[\alpha(y),[z, x]]=0
\end{gathered}
$$

for all homogeneous elements $x, y, z$ in $\mathfrak{L}$.

It is multiplicative if, in addition $\alpha \circ[\cdot, \cdot]=[\cdot, \cdot] \circ \alpha^{\otimes 2}$, (i.e., $\left.\alpha([x, y])=[\alpha(x), \alpha(y)], \forall x, y \in \mathfrak{L}\right)$.

Definition 1.2. ([13], [19]). A Hom-Lie supercoalgebra is a triple $(\mathfrak{L}, \Delta, \alpha)$ consisting of a superspace $\mathfrak{L}$, an even superspace homomorphism $\alpha: \mathfrak{L} \rightarrow \mathfrak{L}$ and a linear map $\Delta: \mathfrak{L} \rightarrow \mathfrak{L} \otimes \mathfrak{L}$ (the cobracket) such that

$$
\begin{gathered}
\Delta\left(\mathfrak{L}^{i}\right) \subset \sum_{i=j+k} \mathfrak{L}^{j} \otimes \mathfrak{L}^{k} \text { for } i \in \mathbb{Z}_{2}, \\
\text { Im } \Delta \subset \operatorname{Im}(1 \otimes 1-\tau) \text { i.e. } \Delta \text { is skew - supersymmetric, } \\
\left(1 \otimes 1 \otimes 1+\xi+\xi^{2}\right) \circ(\alpha \otimes \Delta) \circ \Delta=0: \mathfrak{L} \rightarrow \mathfrak{L} \otimes \mathfrak{L} \otimes \mathfrak{L} .
\end{gathered}
$$

If, in addition, $\Delta \circ \alpha=\alpha^{\otimes 2} \circ \Delta$, then $\mathfrak{L}$ is called co-multiplicative.

A Hom-Lie supercoalgebra with $\alpha=I d$ is exactly a Lie supercoalgebra [13].

Remark 1.3. Let $\operatorname{Im} \Delta \subset \operatorname{Im}(1 \otimes 1-\tau) \subset \operatorname{ker}(1 \otimes 1+\tau)$, then $(1 \otimes 1+\tau) \circ \Delta=0$ i.e. $\Delta$ is skew-supersymmetric ([24]).

Definition 1.4. (1) For an element $x$ in a Hom-Lie superalgebra $(\mathfrak{L},[\cdot, \cdot], \alpha)$ and $n \geq 2$, define the adjoint $\operatorname{map} a d_{x}: \mathfrak{L}^{\otimes n} \rightarrow \mathfrak{L}^{\otimes n}$ by

$$
a d_{x}\left(y_{1} \otimes \cdots \otimes y_{n}\right)=\sum_{i=1}^{n}(-1)^{|x|\left(\left|y_{1}\right|+\left|y_{2}\right|+\cdots+\left|y_{i-1}\right|\right)} \alpha\left(y_{1}\right) \otimes \cdots \otimes \alpha\left(y_{i-1}\right) \otimes\left[x, y_{i}\right] \otimes \alpha\left(y_{i+1}\right) \cdots \otimes \alpha\left(y_{n}\right)
$$

For $n=2, a d_{x}\left(y_{1} \otimes y_{2}\right)=\left[x, y_{1}\right] \otimes \alpha\left(y_{2}\right)+(-1)^{|x|\left|y_{1}\right|} \alpha\left(y_{1}\right) \otimes\left[x, y_{2}\right]$

Conversely, given $\gamma=y_{1} \otimes \cdots \otimes y_{n}$, we define the map $\operatorname{ad}(\gamma): \mathfrak{L} \rightarrow \mathfrak{L}^{\otimes n}$ by $\operatorname{ad}(\gamma)(x)=a d_{x}(\gamma)$, for $x \in \mathfrak{L}$. (2) For an element $x$ in a Hom-Lie superalgebra $(\mathfrak{L},[\cdot, \cdot], \alpha)$ and $n \geq 2$, define the adjoint map $a d_{\alpha(x)}: \mathfrak{L}^{\otimes n} \rightarrow \mathfrak{L}^{\otimes n}$ by

$$
a d_{\alpha(x)}\left(y_{1} \otimes \cdots \otimes y_{n}\right)=\sum_{i=1}^{n}(-1)^{|x|\left(\left|y_{1}\right|+\left|y_{2}\right|+\cdots+\left|y_{i-1}\right|\right)} \alpha\left(y_{1}\right) \otimes \cdots \otimes \alpha\left(y_{i-1}\right) \otimes\left[\alpha(x), y_{i}\right] \otimes \alpha\left(y_{i+1}\right) \cdots \otimes \alpha\left(y_{n}\right)
$$

Definition 1.5. A (multiplicative) Hom-Lie superbialgebra is a quadruple $(\mathfrak{L},[\cdot, \cdot], \Delta, \alpha)$ such that

1. $(\mathfrak{L},[\cdot, \cdot], \alpha)$ is a (multiplicative) Hom-Lie superalgebra.

2. $(\mathfrak{L}, \Delta, \alpha)$ is a (co-multiplicative) Hom-Lie supercoalgebra. 
3. The following compatibility condition holds for all $x, y \in \mathfrak{L}$ :

$$
\Delta([x, y])=a d_{\alpha(x)}(\Delta(y))-(-1)^{|x||y|} a d_{\alpha(y)}(\Delta(x)) .
$$

Definition 1.6. The map $f: \mathfrak{L} \rightarrow \mathfrak{L}^{\prime}$ is called even (resp. odd) map if $f\left(\mathfrak{L}_{i}\right) \subset \mathfrak{L}^{\prime}{ }_{i}\left(\right.$ resp. $\left.f\left(\mathfrak{L}_{i}\right) \subset \mathfrak{L}^{\prime}{ }_{i+1}\right)$, for $i=0,1$. A morphism of Hom-Lie superbialgebras is an even linear map such that

$$
\alpha \circ f=f \circ \alpha, \quad f \circ[\cdot, \cdot]=[\cdot, \cdot] \circ f^{\otimes 2} \quad \text { and } \quad \Delta \circ f=f^{\otimes 2} \circ \Delta .
$$

An isomorphism of Hom-Lie superbialgebras is an invertible morphism of Hom-Lie superbialgebras. Two Hom-Lie superbialgebras are said to be isomorphic if there exists an isomorphism between them.

Remark 1.7. A Hom-Lie superbialgebra with $\alpha=I d$ is exactly a Lie superbialgebra, as defined in [13, 7, 8].

Remark 1.8. The compatibility condition (1.8) is, in fact, a cocycle condition in Hom-Lie superalgebra cohomology [20], just as it is the case in a Lie superbialgebra with Lie superalgebra cohomology $[8]$. Indeed, we can regard $\mathfrak{L}^{\otimes 2}$ as an $\mathfrak{L}$-module via the $\alpha$-twisted adjoint action (1.8):

$$
\begin{aligned}
x \cdot\left(y_{1} \otimes y_{2}\right) & =a d_{\alpha(x)}\left(y_{1} \otimes y_{2}\right) \\
& =\left[\alpha(x), y_{1}\right] \otimes \alpha\left(y_{2}\right)+(-1)^{|x|\left|y_{1}\right|} \alpha\left(y_{1}\right) \otimes\left[\alpha(x), y_{2}\right],
\end{aligned}
$$

for $x \in \mathfrak{L}$ and $y_{1} \otimes y_{2} \in \mathfrak{L}^{\otimes 2}$.

Then we can think of the cobracket $\Delta: \mathfrak{L} \rightarrow \mathfrak{L} \otimes \mathfrak{L}$ as a 1 -cochain $\Delta \in C^{1}\left(\mathfrak{L}, \mathfrak{L}^{\otimes 2}\right)$. Here $C^{1}\left(\mathfrak{L}, \mathfrak{L}^{\otimes 2}\right)$ is defined as the linear super-subspace of $\operatorname{Hom}\left(\mathfrak{L}, \mathfrak{L}^{\otimes 2}\right)$ consisting of maps that commute with $\alpha$. Generalizing [20] to include coefficients in $\mathfrak{L}^{\otimes 2}$, the differential on $\Delta$ is given by

$\left(\delta_{H L}^{1} \Delta\right)(x, y)=\Delta([x, y])-x \cdot \Delta(y)+(-1)^{|x||y|} y \cdot \Delta(x)=\Delta([x, y])-a d_{\alpha(x)}(\Delta(y))+(-1)^{|x||y|} a d_{\alpha(y)}(\Delta(x))$.

Therefore, (1.8) says exactly that $\Delta \in C^{1}\left(\mathfrak{L}, \mathfrak{L}^{\otimes 2}\right)$ is a 1-cocycle.

Example 1.9. Classification of 2-dimensional Hom-Lie superbialgebras with 1-dimensional even part.

Let $\mathfrak{L}=\mathfrak{L}_{\overline{0}} \oplus \mathfrak{L}_{\overline{1}}$ be a 2-dimensional superspace where $\mathfrak{L}_{\overline{0}}$ is generated by $e_{1}$ and $\mathfrak{L}_{\overline{1}}$ is generated by $e_{2}$. The triple $(\mathfrak{L},[\cdot, \cdot], \alpha)$ is a Hom-Lie superalgebra when $\left[e_{1}, e_{1}\right]=0,\left[e_{1}, e_{2}\right]=b e_{2}$ and $\left[e_{2}, e_{2}\right]=c e_{1}$ with $\alpha\left(e_{1}\right)=a_{1} e_{1}, \alpha\left(e_{2}\right)=a_{2} e_{2}$ and $a_{2} b c=0$, where $b, c, a_{1}, a_{2}$ are parameters in $\mathbb{K}$.

The triple $(\mathfrak{L}, \Delta, \alpha)$ is a Hom-Lie supercoalgebra if $\Delta\left(e_{1}\right)=0$ and $\Delta\left(e_{2}\right)=d\left(e_{1} \otimes e_{2}-e_{2} \otimes e_{1}\right)$, where $d \in \mathbb{K}$. The triple $(\mathfrak{L},[\cdot, \cdot], \Delta, \alpha)$ is a Hom-Lie superbialgebras if $a_{1}=1$ or $a_{1}=-1$ and $a_{2} b d=0$.

Remark 1.10. Recently Cai and Sheng introduced a different notion of Hom-Lie bialgebras called purely Hom-Lie bialgebra, see [5], which we can extend to the super case as follows. Let $(\mathfrak{L},[\cdot, \cdot], \alpha)$, where $\alpha$ is invertible, and $\left(\mathfrak{L}^{*},[\cdot, \cdot],\left(\alpha^{-1}\right)^{*}\right)$ be two Hom-Lie superalgebras. The pair $\left(\mathfrak{L}, \mathfrak{L}^{*}\right)$ is a purely Hom-Lie superbialgebra if holds the compatibility condition

$$
\Delta([x, y])=a d_{\alpha^{-1}(x)}(\Delta(y))-(-1)^{|x||y|} a_{\alpha^{-1}(y)}(\Delta(x)) .
$$

Notice that this condition is different from condition (1.8). Purely Hom-Lie bialgebras in a graded case will be studied in a forthcoming paper.

Classification of 3-dimensional Hom-Lie superbialgebras with 2-dimensional even part.

Let $\mathfrak{L}=\mathfrak{L}_{\overline{0}} \oplus \mathfrak{L}_{\overline{1}}$ be a 3 -dimensional superspace where $\mathfrak{L}_{\overline{0}}$ is generated by $e_{1}, e_{2}$ and $\mathfrak{L}_{\overline{1}}$ is generated by $e_{3}$. We aim to construct Hom-Lie superbialgebras. We set for the linear map $\alpha$

$$
\alpha\left(e_{1}\right)=a_{1} e_{1}+a_{2} e_{2}, \quad \alpha\left(e_{2}\right)=a_{3} e_{1}+a_{4} e_{2}, \quad \alpha\left(e_{3}\right)=a_{5} e_{3},
$$

where $a_{1}, a_{2}, a_{3}, a_{4}, a_{5}$ are parameters in $\mathbb{K}$.

The structure of the bracket $[\cdot, \cdot]$ is of the form

$$
\left[e_{3}, e_{3}\right]=b_{1} e_{1}+b_{2} e_{2}, \quad\left[e_{1}, e_{2}\right]=b_{3} e_{1}+b_{4} e_{2}, \quad\left[e_{1}, e_{3}\right]=b_{5} e_{3}, \quad\left[e_{2}, e_{3}\right]=b_{6} e_{3},
$$


where $b_{1}, b_{2}, b_{3}, b_{4}, b_{5}, b_{6}$ are parameters in $\mathbb{K}$.

The structure of the cobracket $\Delta$ is of the form

$$
\begin{gathered}
\Delta\left(e_{1}\right)=c_{1}\left(e_{1} \otimes e_{2}-e_{2} \otimes e_{1}\right)+c_{5} e_{3} \otimes e_{3}, \quad \Delta\left(e_{2}\right)=c_{2}\left(e_{1} \otimes e_{2}-e_{2} \otimes e_{1}\right)+c_{6} e_{3} \otimes e_{3}, \\
\Delta\left(e_{3}\right)=c_{3}\left(e_{1} \otimes e_{3}-e_{3} \otimes e_{1}\right)+c_{4}\left(e_{2} \otimes e_{3}-e_{3} \otimes e_{2}\right),
\end{gathered}
$$

where $c_{1}, c_{2}, c_{3}, c_{4}$ are parameters in $\mathbb{K}$.

In the sequel, we will consider the linear map under the Jordan forms with respect to a suitable basis. We split the calculations in two cases. First, we deal with diagonal case and then with Jordan case.

1) Diagonal case : We consider the linear map $\alpha$, where with respect to a suitable basis the matrix is of the form: $\alpha=\left(\begin{array}{lll}a_{1} & 0 & 0 \\ 0 & a_{4} & 0 \\ 0 & 0 & a_{5}\end{array}\right)$, which corresponds to $a_{2}=0, a_{3}=0$, and the eigenvalues are pairwise non-equal.

We obtained, when the eigenvalues are nonzero, the following corresponding (multiplicative) Hom-Lie su-

\begin{tabular}{|c|c|c|}
\hline Linear map & bracket & cobracket \\
\hline$\alpha\left(e_{1}\right)=e_{1}, \quad \alpha\left(e_{2}\right)=a_{4} e_{2}, \quad \alpha\left(e_{3}\right)=-e_{3}$ & $\begin{array}{l}{\left[e_{3}, e_{3}\right]=0,\left[e_{1}, e_{3}\right]=b_{5} e_{3} \text { or }\left(b_{5}=0\right),} \\
{\left[e_{1}, e_{2}\right]=b_{4} e_{2},\left[e_{2}, e_{3}\right]=0}\end{array}$ & $\begin{array}{l}\Delta\left(e_{1}\right)=c_{5} e_{3} \otimes e_{3} \\
\Delta\left(e_{2}\right)=0 \\
\Delta\left(e_{3}\right)=0\end{array}$ \\
\hline$\alpha\left(e_{1}\right)=e_{1}, \quad \alpha\left(e_{2}\right)=a_{4} e_{2}, \quad \alpha\left(e_{3}\right)=a_{5} e_{3}$ & All bracket are zero & $\begin{array}{l}\Delta\left(e_{1}\right)=0 \\
\Delta\left(e_{2}\right)=c_{2}\left(e_{1} \otimes e_{2}-e_{2} \otimes e_{1}\right) \\
\Delta\left(e_{3}\right)=c_{3}\left(e_{1} \otimes e_{3}-e_{3} \otimes e_{1}\right)\end{array}$ \\
\hline$\alpha\left(e_{1}\right)=e_{1}, \quad \alpha\left(e_{2}\right)=a_{4} e_{2}, \quad \alpha\left(e_{3}\right)=-e_{3}$ & $\begin{array}{l}{\left[e_{3}, e_{3}\right]=b_{1} e_{1},} \\
{\left[e_{1}, e_{2}\right]=\left[e_{1}, e_{3}\right]=\left[e_{2}, e_{3}\right]=0}\end{array}$ & $\begin{array}{l}\Delta\left(e_{1}\right)=0 \\
\Delta\left(e_{2}\right)=c_{2}\left(e_{1} \otimes e_{2}-e_{2} \otimes e_{1}\right) \\
\Delta\left(e_{3}\right)=c_{3}\left(e_{1} \otimes e_{3}-e_{3} \otimes e_{1}\right)\end{array}$ \\
\hline$\alpha\left(e_{1}\right)=a_{1} e_{1}, \quad \alpha\left(e_{2}\right)=e_{2}, \alpha\left(e_{3}\right)=-e_{3}$ & $\begin{array}{l}{\left[e_{3}, e_{3}\right]=\left[e_{1}, e_{3}\right]=0,\left[e_{1}, e_{2}\right]=b_{3} e_{1}} \\
{\left[e_{2}, e_{3}\right]=b_{6} e_{3} \text { or }\left(b_{6}=0 \text { and } b_{3}=0\right)}\end{array}$ & $\begin{array}{l}\Delta\left(e_{1}\right)=0 \\
\Delta\left(e_{2}\right)=c_{6} e_{3} \otimes e_{3} \\
\Delta\left(e_{3}\right)=0\end{array}$ \\
\hline$\alpha\left(e_{1}\right)=a_{1} e_{1}, \quad \alpha\left(e_{2}\right)=e_{2}, \alpha\left(e_{3}\right)=-e_{3}$ & $\begin{array}{l}{\left[e_{1}, e_{2}\right]=\left[e_{1}, e_{3}\right]=\left[e_{2}, e_{3}\right]=0} \\
{\left[e_{3}, e_{3}\right]=b_{2} e_{2} \text { or }\left(b_{2}=0\right)}\end{array}$ & $\begin{array}{l}\Delta\left(e_{1}\right)=c_{1}\left(e_{1} \otimes e_{2}-e_{2} \otimes e_{1}\right) \\
\Delta\left(e_{2}\right)=0 \\
\Delta\left(e_{3}\right)=c_{4}\left(e_{2} \otimes e_{3}-e_{3} \otimes e_{2}\right)\end{array}$ \\
\hline$\alpha\left(e_{1}\right)=e_{1}, \alpha\left(e_{2}\right)=a_{4} e_{2}, \alpha\left(e_{3}\right)=a_{5} e_{3}$ & $\begin{array}{l}{\left[e_{3}, e_{3}\right]=\left[e_{1}, e_{3}\right]=\left[e_{2}, e_{3}\right]=0,} \\
{\left[e_{1}, e_{2}\right]=b_{4} e_{2}}\end{array}$ & $\begin{array}{l}\Delta\left(e_{1}\right)=0 \\
\Delta\left(e_{2}\right)=c_{2}\left(e_{1} \otimes e_{2}-e_{2} \otimes e_{1}\right) \\
\Delta\left(e_{3}\right)=0\end{array}$ \\
\hline$\alpha\left(e_{1}\right)=e_{1}, \alpha\left(e_{2}\right)=a_{4} e_{2}, \alpha\left(e_{3}\right)=a_{5} e_{3}$ & $\begin{array}{l}{\left[e_{3}, e_{3}\right]=\left[e_{2}, e_{3}\right]=0} \\
{\left[e_{1}, e_{2}\right]=b_{4} e_{2},\left[e_{1}, e_{3}\right]=b_{5} e_{3}}\end{array}$ & All cobracket are zero \\
\hline $\begin{array}{l}\alpha\left(e_{1}\right)=a_{1} e_{1}, \quad \alpha\left(e_{2}\right)=a_{4} e_{2}, \quad \alpha\left(e_{3}\right)=a_{5} e_{3} \\
a_{5}= \pm \sqrt{a_{1}} \text { or }\left(\alpha\left(e_{1}\right)=e_{1} \text { and } \alpha\left(e_{3}\right)=-e_{3}\right)\end{array}$ & All bracket are zero & $\begin{array}{l}\Delta\left(e_{1}\right)=c_{5} e_{3} \otimes e_{3} \\
\Delta\left(e_{2}\right)=0 \\
\Delta\left(e_{3}\right)=0\end{array}$ \\
\hline$\alpha\left(e_{1}\right)=a_{1} e_{1}, \alpha\left(e_{2}\right)=e_{2}, \alpha\left(e_{3}\right)=a_{5} e_{3}$ & $\begin{array}{l}{\left[e_{3}, e_{3}\right]=\left[e_{1}, e_{3}\right]=0} \\
{\left[e_{1}, e_{2}\right]=b_{3} e_{1},\left[e_{2}, e_{3}\right]=b_{6} e_{3}}\end{array}$ & All cobracket are zero \\
\hline $\begin{array}{l}\alpha\left(e_{1}\right)=e_{1}, \quad \alpha\left(e_{2}\right)=a_{4} e_{2}, \quad \alpha\left(e_{3}\right)=a_{5} e_{3} \\
\left(a_{5}= \pm \sqrt{a_{4}}\right)\end{array}$ & $\begin{array}{l}{\left[e_{3}, e_{3}\right]=b_{2} e_{2},\left[e_{1}, e_{2}\right]=b_{4} e_{2}} \\
\left(b_{5}=\frac{a_{5} b_{4}}{2 a_{4}}, c_{3}=\frac{a_{5} c_{2}}{2 a_{4}}, c_{6}=-\frac{b_{4} c_{2}}{b_{2}}\right) \\
{\left[e_{2}, e_{3}\right]=0,\left[e_{1}, e_{3}\right]=b_{5} e_{3}}\end{array}$ & $\begin{array}{l}\Delta\left(e_{1}\right)=0, \Delta\left(e_{2}\right)=c_{2}\left(e_{1} \otimes e_{2}-\right. \\
\left.e_{2} \otimes e_{1}\right)+c_{6} e_{3} \otimes e_{3} \\
\Delta\left(e_{3}\right)=c_{3}\left(e_{1} \otimes e_{3}-e_{3} \otimes e_{1}\right)\end{array}$ \\
\hline $\begin{array}{l}\alpha\left(e_{1}\right)=a_{1} e_{1}, \quad \alpha\left(e_{2}\right)=e_{2}, \quad \alpha\left(e_{3}\right)=a_{5} e_{3} \\
\left(a_{5}= \pm \sqrt{a_{1}}\right)\end{array}$ & $\begin{array}{l}{\left[e_{3}, e_{3}\right]=b_{1} e_{1},\left[e_{1}, e_{2}\right]=b_{3} e_{1},} \\
\left(b_{6}=-\frac{a_{5} b_{3}}{2 a_{1}}, c_{4}=-\frac{a_{5} c_{1}}{2 a_{1}}, c_{5}=-\frac{b_{3} c_{1}}{b_{1}}\right) \\
{\left[e_{1}, e_{3}\right]=0,\left[e_{2}, e_{3}\right]=b_{6} e_{3}}\end{array}$ & $\begin{array}{l}\Delta\left(e_{1}\right)=c_{1}\left(e_{1} \otimes e_{2}-e_{2} \otimes e_{1}\right) \\
+c_{5} e_{3} \otimes e_{3}, \Delta\left(e_{2}\right)=0 \\
\Delta\left(e_{3}\right)=c_{4}\left(e_{2} \otimes e_{3}-e_{3} \otimes e_{2}\right)\end{array}$ \\
\hline $\begin{array}{l}\alpha\left(e_{1}\right)=a_{1} e_{1}, \quad \alpha\left(e_{2}\right)=e_{2}, \quad \alpha\left(e_{3}\right)=a_{5} e_{3} \\
\left(a_{5}= \pm \sqrt{a_{1}}\right)\end{array}$ & $\begin{array}{l}{\left[e_{3}, e_{3}\right]=0,\left[e_{1}, e_{2}\right]=b_{3} e_{1}} \\
\left(b_{6}=-\frac{a_{5} b_{3}}{2 a_{1}}\right) \\
{\left[e_{1}, e_{3}\right]=0,\left[e_{2}, e_{3}\right]=b_{6} e_{3}}\end{array}$ & $\begin{array}{l}\Delta\left(e_{1}\right)=c_{5} e_{3} \otimes e_{3} \\
\Delta\left(e_{2}\right)=0 \\
\Delta\left(e_{3}\right)=0\end{array}$ \\
\hline $\begin{array}{l}\alpha\left(e_{1}\right)=a_{1} e_{1}, \quad \alpha\left(e_{2}\right)=e_{2}, \quad \alpha\left(e_{3}\right)=a_{5} e_{3} \\
\left(a_{5}= \pm \sqrt{a_{1}}, c_{4}=-\frac{a_{5} c_{1}}{2 a_{1}}\right)\end{array}$ & All bracket are zero & $\begin{array}{l}\Delta\left(e_{1}\right)=c_{1}\left(e_{1} \otimes e_{2}-e_{2} \otimes e_{1}\right) \\
+c_{5} e_{3} \otimes e_{3}, \Delta\left(e_{2}\right)=0 \\
\Delta\left(e_{3}\right)=c_{4}\left(e_{2} \otimes e_{3}-e_{3} \otimes e_{2}\right)\end{array}$ \\
\hline
\end{tabular}
perbialgebras : 


\begin{tabular}{|c|c|c|}
\hline $\begin{array}{l}\alpha\left(e_{1}\right)=e_{1}, \quad \alpha\left(e_{2}\right)=a_{4} e_{2}, \quad \alpha\left(e_{3}\right)=a_{5} e_{3} \\
\left(a_{5}= \pm \sqrt{a_{4}}, c_{3}=\frac{a_{5} c_{2}}{2 a_{4}}\right)\end{array}$ & All bracket are zero & $\begin{array}{l}\Delta\left(e_{1}\right)=0, \Delta\left(e_{2}\right)=c_{2}\left(e_{1} \otimes e_{2}\right. \\
\left.-e_{2} \otimes e_{1}\right)+c_{6} e_{3} \otimes e_{3} \\
\Delta\left(e_{3}\right)=c_{3}\left(e_{1} \otimes e_{3}-e_{3} \otimes e_{1}\right)\end{array}$ \\
\hline $\begin{array}{l}\alpha\left(e_{1}\right)=e_{1}, \quad \alpha\left(e_{2}\right)=a_{4} e_{2}, \quad \alpha\left(e_{3}\right)=a_{5} e_{3} \\
\left(a_{5}= \pm \sqrt{a_{4}}\right)\end{array}$ & All bracket are zero & $\begin{array}{l}\Delta\left(e_{1}\right)=0 \\
\Delta\left(e_{2}\right)=c_{2}\left(e_{1} \otimes e_{2}-e_{2} \otimes e_{1}\right) \\
\Delta\left(e_{3}\right)=c_{3}\left(e_{1} \otimes e_{3}-e_{3} \otimes e_{1}\right)\end{array}$ \\
\hline $\begin{array}{l}\alpha\left(e_{1}\right)=e_{1}, \alpha\left(e_{2}\right)=a_{4} e_{2}, \alpha\left(e_{3}\right)=a_{5} e_{3} \\
\left(a_{5}= \pm \sqrt{a_{4}}\right)\end{array}$ & $\begin{array}{l}{\left[e_{1}, e_{2}\right]=b_{4} e_{2},} \\
{\left[e_{3}, e_{3}\right]=\left[e_{1}, e_{3}\right]=\left[e_{2}, e_{3}\right]=0}\end{array}$ & $\begin{array}{l}\Delta\left(e_{1}\right)=0 \\
\Delta\left(e_{2}\right)=c_{2}\left(e_{1} \otimes e_{2}-e_{2} \otimes e_{1}\right) \\
\Delta\left(e_{3}\right)=0\end{array}$ \\
\hline $\begin{array}{l}\alpha\left(e_{1}\right)=e_{1}, \quad \alpha\left(e_{2}\right)=a_{4} e_{2}, \quad \alpha\left(e_{3}\right)=a_{5} e_{3} \\
\left(a_{5}= \pm \sqrt{a_{4}}\right)\end{array}$ & $\begin{array}{l}{\left[e_{1}, e_{2}\right]=b_{4} e_{2},\left[e_{1}, e_{3}\right]=b_{5} e_{3},} \\
\left(b_{5}=\frac{a_{5} b_{4}}{2 a_{4}}\right) \\
{\left[e_{2}, e_{3}\right]=\left[e_{3}, e_{3}\right]=0}\end{array}$ & $\begin{array}{l}\Delta\left(e_{1}\right)=0 \\
\Delta\left(e_{2}\right)=c_{6} e_{3} \otimes e_{3} \\
\Delta\left(e_{3}\right)=0\end{array}$ \\
\hline$\alpha\left(e_{1}\right)=a_{1} e_{1}, \alpha\left(e_{2}\right)=a_{4} e_{2}, \alpha\left(e_{3}\right)=a_{5} e_{3}$ & All bracket are zero & All cobracket are zero \\
\hline $\begin{array}{l}\alpha\left(e_{1}\right)=a_{1} e_{1}, \alpha\left(e_{2}\right)=e_{2}, \alpha\left(e_{3}\right)=a_{5} e_{3} \\
\left(a_{5}= \pm \sqrt{a_{1}}\right)\end{array}$ & $\begin{array}{l}{\left[e_{1}, e_{2}\right]=b_{3} e_{1},} \\
{\left[e_{1}, e_{3}\right]=\left[e_{2}, e_{3}\right]=\left[e_{3}, e_{3}\right]=0}\end{array}$ & $\begin{array}{l}\Delta\left(e_{1}\right)=c_{1}\left(e_{1} \otimes e_{2}-e_{2} \otimes e_{1}\right) \\
\Delta\left(e_{2}\right)=0 \\
\Delta\left(e_{3}\right)=0\end{array}$ \\
\hline $\begin{array}{l}\alpha\left(e_{1}\right)=a_{1} e_{1}, \alpha\left(e_{2}\right)=a_{4} e_{2}, \alpha\left(e_{3}\right)=a_{5} e_{3} \\
\left(a_{5}= \pm \sqrt{a_{1}}\right)\end{array}$ & $\begin{array}{l}{\left[e_{3}, e_{3}\right]=b_{1} e_{1}} \\
{\left[e_{1}, e_{2}\right]=\left[e_{1}, e_{3}\right]=\left[e_{2}, e_{3}\right]=0}\end{array}$ & All cobracket are zero \\
\hline $\begin{array}{l}\alpha\left(e_{1}\right)=a_{1} e_{1}, \alpha\left(e_{2}\right)=a_{4} e_{2}, \alpha\left(e_{3}\right)=a_{5} e_{3} \\
\left(a_{5}= \pm \sqrt{a_{4}}\right)\end{array}$ & $\begin{array}{l}{\left[e_{3}, e_{3}\right]=b_{2} e_{2},} \\
{\left[e_{1}, e_{2}\right]=\left[e_{1}, e_{3}\right]=\left[e_{2}, e_{3}\right]=0}\end{array}$ & All cobracket are zero \\
\hline$\alpha\left(e_{1}\right)=e_{1}, \quad \alpha\left(e_{2}\right)=a_{4} e_{2}, \quad \alpha\left(e_{3}\right)=-e_{3}$ & All bracket are zero & $\begin{array}{l}\Delta\left(e_{1}\right)=0 \\
\Delta\left(e_{2}\right)=0 \\
\Delta\left(e_{3}\right)=c_{3}\left(e_{1} \otimes e_{3}-e_{3} \otimes e_{1}\right)\end{array}$ \\
\hline$\alpha\left(e_{1}\right)=a_{1} e_{1}, \quad \alpha\left(e_{2}\right)=e_{2}, \quad \alpha\left(e_{3}\right)=-e_{3}$ & All bracket are zero & $\begin{aligned} \Delta\left(e_{1}\right) & =0 \\
\Delta\left(e_{2}\right) & =0 \\
\Delta\left(e_{3}\right) & =c_{4}\left(e_{2} \otimes e_{3}-e_{3} \otimes e_{2}\right)\end{aligned}$ \\
\hline$\alpha\left(e_{1}\right)=e_{1}, \quad \alpha\left(e_{2}\right)=a_{4} e_{2}, \quad \alpha\left(e_{3}\right)=-e_{3}$ & $\begin{array}{l}{\left[e_{1}, e_{2}\right]=b_{4} e_{2},} \\
{\left[e_{1}, e_{3}\right]=\left[e_{2}, e_{3}\right]=\left[e_{3}, e_{3}\right]=0}\end{array}$ & $\begin{array}{l}\Delta\left(e_{1}\right)=0 \\
\Delta\left(e_{2}\right)=c_{2}\left(e_{1} \otimes e_{2}-e_{2} \otimes e_{1}\right) \\
\Delta\left(e_{3}\right)=0\end{array}$ \\
\hline
\end{tabular}

2) Jordan case : Now, we consider the linear map $\alpha$ where the corresponding matrix is of the form $\alpha=\left(\begin{array}{ccc}a_{1} & 1 & 0 \\ 0 & a_{1} & 0 \\ 0 & 0 & a_{5}\end{array}\right)$, that is $a_{2}=1, a_{3}=0, a_{4}=a_{1}$.

We obtained the following corresponding (multiplicative) Hom-Lie superbialgebras :

\begin{tabular}{|c|c|c|}
\hline Linear map & bracket & cobracket \\
\hline$\alpha\left(e_{1}\right)=e_{2}, \quad \alpha\left(e_{2}\right)=\alpha\left(e_{3}\right)=0$ & $\begin{array}{l}{\left[e_{3}, e_{3}\right]=b_{2} e_{2},\left[e_{1}, e_{2}\right]=b_{4} e_{2}} \\
{\left[e_{1}, e_{3}\right]=\left[e_{2}, e_{3}\right]=0}\end{array}$ & $\begin{array}{l}\Delta\left(e_{1}\right)=c_{1}\left(e_{1} \otimes e_{2}-e_{2} \otimes e_{1}\right) \\
+c_{5} e_{3} \otimes e_{3}, \Delta\left(e_{2}\right)=0 \\
\Delta\left(e_{3}\right)=c_{4}\left(e_{2} \otimes e_{3}-e_{3} \otimes e_{2}\right)\end{array}$ \\
\hline$\alpha\left(e_{1}\right)=e_{2}, \quad \alpha\left(e_{2}\right)=\alpha\left(e_{3}\right)=0$ & $\begin{array}{l}{\left[e_{3}, e_{3}\right]=b_{2} e_{2},\left[e_{1}, e_{2}\right]=b_{4} e_{2},} \\
{\left[e_{1}, e_{3}\right]=b_{5} e_{3},\left[e_{2}, e_{3}\right]=0, \text { or }\left(b_{5}=0\right)}\end{array}$ & $\begin{array}{l}\Delta\left(e_{1}\right)=c_{1}\left(e_{1} \otimes e_{2}-e_{2} \otimes e_{1}\right) \\
+c_{5} e_{3} \otimes e_{3}, \Delta\left(e_{2}\right)=0 \\
\Delta\left(e_{3}\right)=0\end{array}$ \\
\hline$\alpha\left(e_{1}\right)=e_{1}+e_{2}, \alpha\left(e_{2}\right)=e_{2}, \quad \alpha\left(e_{3}\right)=0$ & $\begin{array}{l}{\left[e_{3}, e_{3}\right]=\left[e_{2}, e_{3}\right]=0} \\
{\left[e_{1}, e_{2}\right]=b_{4} e_{2},\left[e_{1}, e_{3}\right]=b_{5} e_{3} \text { or }\left(b_{5}=0\right)}\end{array}$ & $\begin{array}{l}\Delta\left(e_{1}\right)=c_{1}\left(e_{1} \otimes e_{2}-e_{2} \otimes e_{1}\right), \\
\Delta\left(e_{2}\right)=0, \\
\Delta\left(e_{3}\right)=c_{4}\left(e_{2} \otimes e_{3}-e_{3} \otimes e_{2}\right)\end{array}$ \\
\hline $\begin{array}{l}\alpha\left(e_{1}\right)=e_{2}, \alpha\left(e_{2}\right)=0, \alpha\left(e_{3}\right)=a_{5} e_{3}, \\
\left(a_{5} \neq 0\right)\end{array}$ & $\begin{array}{l}{\left[e_{3}, e_{3}\right]=\left[e_{1}, e_{3}\right]=\left[e_{2}, e_{3}\right]=0} \\
{\left[e_{1}, e_{2}\right]=b_{4} e_{2}}\end{array}$ & $\begin{array}{l}\Delta\left(e_{1}\right)=c_{1}\left(e_{1} \otimes e_{2}-e_{2} \otimes e_{1}\right) \\
\Delta\left(e_{2}\right)=\Delta\left(e_{3}\right)=0\end{array}$ \\
\hline$\alpha\left(e_{1}\right)=e_{1}+e_{2}, \quad \alpha\left(e_{2}\right)=e_{2}, \alpha\left(e_{3}\right)=a_{5} e_{3}$ & All bracket are zero & $\begin{array}{l}\Delta\left(e_{1}\right)=c_{1}\left(e_{1} \otimes e_{2}-e_{2} \otimes e_{1}\right), \\
\Delta\left(e_{2}\right)=0, \\
\Delta\left(e_{3}\right)=c_{4}\left(e_{2} \otimes e_{3}-e_{3} \otimes e_{2}\right)\end{array}$ \\
\hline$\alpha\left(e_{1}\right)=e_{1}+e_{2}, \alpha\left(e_{2}\right)=e_{2}, \alpha\left(e_{3}\right)=a_{5} e_{3}$ & $\begin{array}{l}{\left[e_{1}, e_{2}\right]=b_{4} e_{2},\left[e_{1}, e_{3}\right]=b_{5} e_{3},} \\
{\left[e_{3}, e_{3}\right]=\left[e_{2}, e_{3}\right]=0}\end{array}$ & $\begin{array}{l}\Delta\left(e_{1}\right)=c_{1}\left(e_{1} \otimes e_{2}-e_{2} \otimes e_{1}\right), \\
\Delta\left(e_{2}\right)=0, \text { for } c_{4}=\frac{b_{5} c_{1}}{b_{4}} \\
\Delta\left(e_{3}\right)=c_{4}\left(e_{2} \otimes e_{3}-e_{3} \otimes e_{2}\right)\end{array}$ \\
\hline $\begin{array}{l}\alpha\left(e_{1}\right)=e_{1}+e_{2}, \quad \alpha\left(e_{2}\right)=e_{2}, \alpha\left(e_{3}\right)=a_{5} e_{3} \\
\left(a_{5}= \pm 1, c_{4}=-\frac{a_{5} c_{1}}{2}\right)\end{array}$ & All bracket are zero & $\begin{array}{l}\Delta\left(e_{1}\right)=c_{1}\left(e_{1} \otimes e_{2}-e_{2} \otimes e_{1}\right) \\
+c_{5} e_{3} \otimes e_{3}, \Delta\left(e_{2}\right)=0 \\
\Delta\left(e_{3}\right)=c_{4}\left(e_{2} \otimes e_{3}-e_{3} \otimes e_{2}\right)\end{array}$ \\
\hline
\end{tabular}




\begin{tabular}{|c|c|c|}
\hline $\begin{array}{l}\alpha\left(e_{1}\right)=e_{1}+e_{2}, \quad \alpha\left(e_{2}\right)=e_{2}, \quad \alpha\left(e_{3}\right)=a_{5} e_{3} \\
\left(a_{5}= \pm 1, c_{4}=-\frac{a_{5} c_{1}}{2}\right)\end{array}$ & $\begin{array}{l}{\left[e_{3}, e_{3}\right]=\left[e_{2}, e_{3}\right]=0} \\
\left(b_{5}=-\frac{a_{5} b_{4}}{2}\right) \\
{\left[e_{1}, e_{2}\right]=b_{4} e_{2},\left[e_{1}, e_{3}\right]=b_{5} e_{3}}\end{array}$ & $\begin{array}{l}\Delta\left(e_{1}\right)=c_{1}\left(e_{1} \otimes e_{2}-e_{2} \otimes e_{1}\right) \\
+c_{5} e_{3} \otimes e_{3}, \Delta\left(e_{2}\right)=0 \\
\Delta\left(e_{3}\right)=c_{4}\left(e_{2} \otimes e_{3}-e_{3} \otimes e_{2}\right)\end{array}$ \\
\hline $\begin{array}{l}\alpha\left(e_{1}\right)=e_{1}+e_{2}, \quad \alpha\left(e_{2}\right)=e_{2}, \quad \alpha\left(e_{3}\right)=a_{5} e_{3} \\
\left(a_{5}= \pm 1\right)\end{array}$ & $\begin{array}{l}{\left[e_{1}, e_{2}\right]=\left[e_{1}, e_{3}\right]=\left[e_{2}, e_{3}\right]=0} \\
{\left[e_{3}, e_{3}\right]=b_{2} e_{2}}\end{array}$ & $\begin{array}{l}\Delta\left(e_{1}\right)=c_{1}\left(e_{1} \otimes e_{2}-e_{2} \otimes e_{1}\right) \\
\Delta\left(e_{2}\right)=0 \\
\Delta\left(e_{3}\right)=c_{4}\left(e_{2} \otimes e_{3}-e_{3} \otimes e_{2}\right)\end{array}$ \\
\hline$\alpha\left(e_{1}\right)=-e_{1}+e_{2}, \alpha\left(e_{2}\right)=-e_{2}, \alpha\left(e_{3}\right)=0$ & $\begin{array}{l}{\left[e_{3}, e_{3}\right]=\left[e_{1}, e_{2}\right]=\left[e_{2}, e_{3}\right]=0} \\
{\left[e_{1}, e_{3}\right]=b_{5} e_{3}, \text { or }\left(b_{5}=0\right)}\end{array}$ & $\begin{array}{l}\Delta\left(e_{1}\right)=\Delta\left(e_{2}\right)=0 \\
\Delta\left(e_{3}\right)=c_{4}\left(e_{2} \otimes e_{3}-e_{3} \otimes e_{2}\right)\end{array}$ \\
\hline $\begin{array}{l}\alpha\left(e_{1}\right)=a_{1} e_{1}+e_{2}, \alpha\left(e_{2}\right)=a_{4} e_{2}, \alpha\left(e_{3}\right)=a_{5} e_{3} \\
\left(a_{1} \neq 0, a_{5} \neq 0\right)\end{array}$ & All bracket are zero & All cobracket are zero \\
\hline $\begin{array}{l}\alpha\left(e_{1}\right)=e_{1}+e_{2}, \quad \alpha\left(e_{2}\right)=e_{2}, \quad \alpha\left(e_{3}\right)=a_{5} e_{3} \\
\left(a_{5}= \pm 1\right)\end{array}$ & $\begin{array}{l}{\left[e_{3}, e_{3}\right]=\left[e_{2}, e_{3}\right]=0,} \\
{\left[e_{1}, e_{3}\right]=b_{5} e_{3},\left[e_{1}, e_{2}\right]=b_{4} e_{2}, \text { or }\left(b_{4}=0\right)}\end{array}$ & $\begin{array}{l}\Delta\left(e_{1}\right)=c_{5} e_{3} \otimes e_{3} \\
\Delta\left(e_{2}\right)=\Delta\left(e_{3}\right)=0\end{array}$ \\
\hline $\begin{array}{l}\alpha\left(e_{1}\right)=e_{1}+e_{2}, \quad \alpha\left(e_{2}\right)=e_{2}, \quad \alpha\left(e_{3}\right)=a_{5} e_{3} \\
\left(a_{5}= \pm 1\right)\end{array}$ & $\begin{array}{l}{\left[e_{3}, e_{3}\right]=b_{2} e_{2},\left[e_{1}, e_{2}\right]=b_{4} e_{2},} \\
\left(b_{5}=\frac{a_{5} b_{4}}{2}, c_{5}=\frac{-b_{4} c_{1}+2 a_{5} b_{4} c_{4}}{2 b_{2}}, c_{4}= \pm \frac{c_{1}}{2}\right) \\
{\left[e_{1}, e_{3}\right]=b_{5} e_{3},\left[e_{2}, e_{3}\right]=0,}\end{array}$ & $\begin{array}{l}\Delta\left(e_{1}\right)=c_{1}\left(e_{1} \otimes e_{2}-e_{2} \otimes e_{1}\right) \\
+c_{5} e_{3} \otimes e_{3}, \Delta\left(e_{2}\right)=0 \\
\Delta\left(e_{3}\right)=c_{4}\left(e_{2} \otimes e_{3}-e_{3} \otimes e_{2}\right)\end{array}$ \\
\hline $\begin{array}{l}\alpha\left(e_{1}\right)=a_{1} e_{1}+e_{2}, \quad \alpha\left(e_{2}\right)=a_{4} e_{2}, \alpha\left(e_{3}\right)=0 \\
\left(a_{1}=a_{4}\right)\end{array}$ & All bracket are zero & $\begin{array}{l}\Delta\left(e_{1}\right)=\Delta\left(e_{2}\right)=0 \\
\Delta\left(e_{3}\right)=c_{4}\left(e_{2} \otimes e_{3}-e_{3} \otimes e_{2}\right)\end{array}$ \\
\hline $\begin{array}{l}\alpha\left(e_{1}\right)=e_{1}+e_{2}, \alpha\left(e_{2}\right)=e_{2}, \alpha\left(e_{3}\right)=a_{5} e_{3} \\
\left(a_{5}= \pm 1\right)\end{array}$ & $\begin{array}{l}{\left[e_{1}, e_{3}\right]=b_{5} e_{3}} \\
{\left[e_{3}, e_{3}\right]=\left[e_{1}, e_{2}\right]=\left[e_{2}, e_{3}\right]=0}\end{array}$ & $\begin{array}{l}\Delta\left(e_{1}\right)=\Delta\left(e_{2}\right)=0 \\
\Delta\left(e_{3}\right)=c_{4}\left(e_{2} \otimes e_{3}-e_{3} \otimes e_{2}\right)\end{array}$ \\
\hline $\begin{array}{l}\alpha\left(e_{1}\right)=a_{1} e_{1}+e_{2}, \alpha\left(e_{2}\right)=a_{4} e_{2}, \alpha\left(e_{3}\right)=a_{5} e_{3} \\
\left(a_{5}= \pm \sqrt{a_{1}}, a_{1}=a_{4}\right)\end{array}$ & $\begin{array}{l}{\left[e_{3}, e_{3}\right]=b_{2} e_{2}} \\
{\left[e_{1}, e_{2}\right]=\left[e_{1}, e_{3}\right]=\left[e_{2}, e_{3}\right]=0}\end{array}$ & All cobracket are zero \\
\hline $\begin{array}{l}\alpha\left(e_{1}\right)=a_{1} e_{1}+e_{2}, \alpha\left(e_{2}\right)=a_{4} e_{2}, \alpha\left(e_{3}\right)=0 \\
\left(a_{1}=a_{4}\right)\end{array}$ & $\begin{array}{l}{\left[e_{1}, e_{3}\right]=b_{5} e_{3}} \\
{\left[e_{1}, e_{2}\right]=\left[e_{3}, e_{3}\right]=\left[e_{2}, e_{3}\right]=0}\end{array}$ & All cobracket are zero \\
\hline
\end{tabular}

The following result shows that a Hom-Lie superbialgebra deforms into another Hom-Lie superbialgebra along any endomorphism.

Theorem 1.11. Let $(\mathfrak{L},[\cdot, \cdot], \Delta, \alpha)$ be a Hom-Lie superbialgebra and an even map $\beta: \mathfrak{L} \rightarrow \mathfrak{L}$ be a Hom-Lie superbialgebra morphism. Then

$$
\mathfrak{L}_{\beta}=\left(\mathfrak{L},[\cdot, \cdot]_{\beta}=\beta \circ[\cdot, \cdot], \Delta_{\beta}=\Delta \circ \beta, \beta \alpha\right)
$$

is also a Hom-Lie superbialgebra, which is multiplicative if $\mathfrak{L}$ is.

Proof. It is immediate that $[\cdot, \cdot]_{\beta}$ is skew-supersymmetric (1.1) because

$$
[x, y]_{\beta}=\beta([x, y])=\beta\left(-(-1)^{|x||y|}[y, x]\right)=-(-1)^{|x||y|} \beta([y, x])=-(-1)^{|x||y|}[y, x]_{\beta} .
$$

The Hom super-Jacobi identity holds in $\mathfrak{L}_{\beta}$ because

$(-1)^{|x||z|}\left[\beta \alpha(x),[y, z]_{\beta}\right]_{\beta}=(-1)^{|x||z|} \beta^{2}[\alpha(x),[y, z]]=-(-1)^{|z||y|}\left[\beta \alpha(z),[x, y]_{\beta}\right]_{\beta}-(-1)^{|y||x|}\left[\beta \alpha(y),[z, x]_{\beta}\right]_{\beta}$,

i.e.,

$$
(-1)^{|x||z|}\left[\beta \alpha(x),[y, z]_{\beta}\right]_{\beta}+(-1)^{|z||y|}\left[\beta \alpha(z),[x, y]_{\beta}\right]_{\beta}+(-1)^{|y||x|}\left[\beta \alpha(y),[z, x]_{\beta}\right]_{\beta}=0 .
$$

$\Delta_{\beta}$ is skew-supersymmetric because

$$
\tau \circ \Delta_{\beta}=\tau \circ \Delta \circ \beta=-\Delta \circ \beta=-\Delta_{\beta} .
$$

Likewise, the Hom-super-co-jacobi identity holds in $\mathfrak{L}_{\beta}$ because

$$
\left(1 \otimes 1 \otimes 1+\xi+\xi^{2}\right) \circ\left(\beta \alpha \otimes \Delta_{\beta}\right) \circ \Delta_{\beta}=\left(\beta^{\otimes 3}\right)^{2}\left(1 \otimes 1 \otimes 1+\xi+\xi^{2}\right) \circ(\alpha \otimes \Delta) \circ \Delta=0 .
$$


To check the compatibility condition (1.8) in $\mathfrak{L}_{\beta}$, we compute as follows :

$$
\begin{aligned}
\Delta_{\beta}\left([x, y]_{\beta}\right) & =\left(\beta^{\otimes 2}\right)^{2} \Delta([x, y]) \\
& =\left(\beta^{\otimes 2}\right)^{2}\left(\left[\alpha(x), y_{1}\right] \otimes \alpha\left(y_{2}\right)\right)+\left(\beta^{\otimes 2}\right)^{2}\left((-1)^{|x|\left|y_{1}\right|} \alpha\left(y_{1}\right) \otimes\left[\alpha(x), y_{2}\right]\right) \\
& -\left(\beta^{\otimes 2}\right)^{2}\left((-1)^{|x| y \mid}\left[\alpha(y), x_{1}\right] \otimes \alpha\left(x_{2}\right)\right)-\left(\beta^{\otimes 2}\right)^{2}\left((-1)^{|x||y|}(-1)^{|y|\left|x_{1}\right|} \alpha\left(x_{1}\right) \otimes\left[\alpha(y), x_{2}\right]\right) \\
& =\left[\beta \alpha(x), \beta\left(y_{1}\right)\right]_{\beta} \otimes \beta \alpha\left(\beta\left(y_{2}\right)\right)+(-1)^{|x|\left|y_{1}\right|} \beta \alpha\left(\beta\left(y_{1}\right)\right) \otimes\left[\beta \alpha(x), \beta\left(y_{2}\right)\right]_{\beta} \\
& -(-1)^{|x||y|}\left[\beta \alpha(y), \beta\left(x_{1}\right)\right]_{\beta} \otimes \beta \alpha\left(\beta\left(x_{2}\right)\right)-(-1)^{|x||y|}(-1)^{|y|\left|x_{1}\right|} \beta \alpha\left(\beta\left(x_{1}\right)\right) \otimes\left[\beta \alpha(y), \beta\left(x_{2}\right)\right]_{\beta} \\
& =\left[\beta \alpha(x), \beta\left(y_{1}\right)\right]_{\beta} \otimes \beta \alpha\left(\beta\left(y_{2}\right)\right)+(-1)^{|x|\left|\beta\left(y_{1}\right)\right|} \beta \alpha\left(\beta\left(y_{1}\right)\right) \otimes\left[\beta \alpha(x), \beta\left(y_{2}\right)\right]_{\beta} \\
& -(-1)^{|x||y|}\left[\beta \alpha(y), \beta\left(x_{1}\right)\right]_{\beta} \otimes \beta \alpha\left(\beta\left(x_{2}\right)\right)-(-1)^{|x||y|}(-1)^{|y|\left|\beta\left(x_{1}\right)\right|} \beta \alpha\left(\beta\left(x_{1}\right)\right) \otimes\left[\beta \alpha(y), \beta\left(x_{2}\right)\right]_{\beta} \\
& =a d_{\beta \alpha(x)}\left(\Delta_{\beta}(y)\right)-(-1)^{|x||y|} a d_{\beta \alpha(y)}\left(\Delta_{\beta}(x)\right) .
\end{aligned}
$$

Because $\left|\beta\left(x_{1}\right)\right|=\left|x_{1}\right|$, and $\left|\beta\left(y_{1}\right)\right|=\left|y_{1}\right|$ (i.e., an even map $\beta$ ). We have shown that $\mathfrak{L}_{\beta}$ is a Hom-Lie superbialgebra. The super-multiplicativity assertion is obvious.

Now we discuss two special cases of Theorem 1.11. The next result says that one can obtain multiplicative Hom-Lie superbialgebras from Lie superbialgebras and their endomorphisms. A construction result of this form for Hom-type algebras was first given in [26].

Corollary 1.12. Let $(\mathfrak{L},[\cdot, \cdot], \Delta)$ be a Lie superbialgebra and an even map $\beta: \mathfrak{L} \rightarrow \mathfrak{L}$ be a Lie superbialgebra morphism. Then

$$
\mathfrak{L}_{\beta}=\left(\mathfrak{L},[\cdot, \cdot]_{\beta}=\beta \circ[\cdot, \cdot], \Delta_{\beta}=\Delta \circ \beta, \beta\right)
$$

is a multiplicative Hom-Lie superbialgebra.

Proof. This is the $\alpha=I d$ special case of Theorem 1.11.

The next result says that every multiplicative Hom-Lie superbialgebra gives rise to an infinite sequence of multiplicative Hom-Lie superbialgebras.

Corollary 1.13. Let $(\mathfrak{L},[\cdot, \cdot], \Delta, \alpha)$ be a multiplicative Hom-Lie superbialgebra. Then

$$
\mathfrak{L}_{\alpha^{n}}=\left(\mathfrak{L},[\cdot, \cdot]_{\alpha^{n}}=\alpha^{n} \circ[\cdot, \cdot], \Delta_{\alpha^{n}}=\Delta \circ \alpha^{n}, \alpha^{n+1}\right)
$$

is also a multiplicative Hom-Lie superbialgebra for each integer $n \geq 0$.

Proof. This is the $\beta=\alpha^{n}$ special case of Theorem 1.11 .

Next we consider when Hom-Lie superbialgebra of the from $\mathfrak{L}_{\beta}$, as in Corollary 1.12, are isomorphic.

Theorem 1.14. Let $\mathfrak{g}$ and $\mathfrak{h}$ be Lie superbialgebras. Let $\alpha: \mathfrak{g} \rightarrow \mathfrak{g}$ and $\beta: \mathfrak{h} \rightarrow \mathfrak{h}$ be Lie superbialgebras morphisms with $\beta$ and $\beta^{\otimes 2}$ injective. Then the following statements are equivalent:

1) The Hom-Lie superbialgebras $\mathfrak{g}_{\alpha}$ and $\mathfrak{h}_{\beta}$ as in Corollary 1.12, are isomorphic.

2) There exists a Lie superbialgebra isomorphism $\gamma: \mathfrak{g} \rightarrow \mathfrak{h}$ such that $\gamma \alpha=\beta \gamma$.

Proof. To show that the first statement implies the second statement, suppose that $\gamma: \mathfrak{g}_{\alpha} \rightarrow \mathfrak{h}_{\beta}$ is an isomorphism of Hom-Lie superbialgebras. Then $\gamma \alpha=\beta \gamma$ automatically.

To see that $\gamma$ is a Lie superbialgebra isomorphism, first we check that it commutes with the Lie bracket. For any two elements $x$ and $y$ in $\mathfrak{g}$, we have

$$
\beta \gamma[x, y]=\gamma \alpha[x, y]=\gamma\left([x, y]_{\alpha}\right)=[\gamma(x), \gamma(y)]_{\beta}=\beta[\gamma(x), \gamma(y)] .
$$

Since $\beta$ is injective, we conclude that $\gamma[x, y]=[\gamma(x), \gamma(y)]$, i.e., $\gamma$ is a Lie superbialgebra isomorphism. To check that $\gamma$ commutes with the Lie cobrackets, we compute as follows:

$$
\begin{aligned}
\beta^{\otimes 2}\left(\gamma^{\otimes 2}(\Delta(x))\right) & =(\beta \gamma)^{\otimes 2}(\Delta(x))=(\gamma \alpha)^{\otimes 2}(\Delta(x))=\gamma^{\otimes 2}\left(\alpha^{\otimes 2}(\Delta(x))\right)=\gamma^{\otimes 2}\left(\Delta_{\alpha}(x)\right)=\Delta_{\beta}(\gamma(x)) \\
& =\beta^{\otimes 2}(\Delta(\gamma(x))) .
\end{aligned}
$$

The injectivity of $\beta^{\otimes 2}$ now implies that $\gamma$ commutes with the Lie cobrackets. Therefore, $\gamma$ is a Lie superbialgebra isomorphism. The other implication is proved by a similar argument, much of which is already given above. 
For a Lie superbialgebra $\mathfrak{g}$, let $\operatorname{Aut}(\mathfrak{g})$ be the group of Lie superbialgebra isomorphisms from $\mathfrak{g}$ to $\mathfrak{g}$. In Theorem 1.14 restricting to the case $\mathfrak{g}=\mathfrak{h}$ with $\alpha$ and $\beta$ both invertible, we obtain the following special case.

Corollary 1.15. Let $\mathfrak{g}$ be a Lie superbialgebra and $\alpha, \beta \in$ Aut $(\mathfrak{g})$. Then the Hom-Lie superbialgebras $\mathfrak{g}_{\alpha}$ and $\mathfrak{g}_{\beta}$, as in Corollary 1.12, are isomorphic if and only if $\alpha$ and $\beta$ are conjugate in Aut $(\mathfrak{g})$.

Corollary 1.15 can be restated as follows.

Corollary 1.16. Let $\mathfrak{g}$ be a Lie superbialgebra. Then there is a bijection between the following two sets:

1) The set of isomorphism classes of Hom-Lie superbialgebras $\mathfrak{g}_{\alpha}$ with $\alpha$ invertible.

2) The set of conjugacy classes in the group Aut $(\mathfrak{g})$.

The next result shows that finite dimensional Hom-Lie superbialgebras, like Lie superbialgebras, can be dualized. A proof of this self-dual property for the special of Lie bialgebras can be found in [16].

Remark 1.17. • If $(\mathfrak{L}, \Delta, \alpha)$ is a Hom-Lie supercoalgebra, then $\left(\mathfrak{L}^{*},[\cdot, \cdot], \alpha\right)$ is a Hom-Lie superalgebra. Here $[\cdot, \cdot]$ and $\alpha$ in $\mathfrak{L}^{*}$ are dual to $\Delta$ and $\alpha$, respectively, in $\mathfrak{L}$.

- Conversely, if $(\mathfrak{L},[\cdot, \cdot], \alpha)$ is a finite dimensional Hom-Lie superalgebra, then $\left(\mathfrak{L}^{*}, \Delta, \alpha\right)$ is a Hom-Lie supercoalgebra, where $\Delta$ and $\alpha$ in $\mathfrak{L}^{*}$ are dual to $[\cdot, \cdot]$ and $\alpha$, respectively, in $\mathfrak{L}$.

Theorem 1.18. Let $(\mathfrak{L},[\cdot, \cdot], \Delta, \alpha)$ be a finite dimensional (multiplicative) Hom-Lie superbialgebra. Then its linear dual $\mathfrak{L}^{*}=\operatorname{Hom}(\mathfrak{L}, \mathbb{K})$ is also a (multiplicative) Hom-Lie superbialgebra with the dual structure maps:

$$
\alpha(\phi)=\phi \circ \alpha, \quad\langle[\phi, \psi], x\rangle=\langle\phi \otimes \psi, \Delta(x)\rangle, \quad\langle\Delta(\phi), x \otimes y\rangle=\langle\phi,[x, y]\rangle,
$$

for $x, y \in \mathfrak{L}$ and $\phi, \psi \in \mathfrak{L}^{*}$.

Proof. As we mentioned right after Remark 1.17, $\left(\mathfrak{L}^{*},[\cdot, \cdot], \alpha\right)$ is a Hom-Lie superalgebra, which is true even if $\mathfrak{L}$ is not finite dimensional. Moreover, $\left(\mathfrak{L}^{*}, \Delta, \alpha\right)$ is a Hom-Lie supercoalgebra, whose validity depends on the finite dimensionality of $\mathfrak{L}$. Thus, it remains to check the compatibility condition (1.8) between the bracket and the cobracket in $\mathfrak{L}^{*}$, i.e.,

$$
\langle\Delta([\phi, \psi]), x \otimes y\rangle=\left\langle a d_{\alpha(\phi)}(\Delta(\psi))-(-1)^{|\phi||\psi|} a d_{\alpha(\psi)}(\Delta(\phi)), x \otimes y\right\rangle
$$

for $x, y \in \mathfrak{L}$ and $\phi, \psi \in \mathfrak{L}^{*}$.

Using Definition 1.12, the compatibility condition (1.8) in $\mathfrak{L}$, we compute the left-hand side of (1.13) as follows:

$$
\begin{aligned}
\langle\Delta([\phi, \psi]), x \otimes y\rangle & =\langle[\phi, \psi],[x, y]\rangle \\
& =\langle\phi \otimes \psi, \Delta([x, y])\rangle \\
& =\left\langle\phi \otimes \psi, a d_{\alpha(x)}(\Delta(y))-(-1)^{|x||y|} a d_{\alpha(y)}(\Delta(x))\right\rangle \\
& =\left\langle\phi \otimes \psi,\left[\alpha(x), y_{1}\right] \otimes \alpha\left(y_{2}\right)\right\rangle+(-1)^{|x|\left|y_{1}\right|}\left\langle\phi \otimes \psi, \alpha\left(y_{1}\right) \otimes\left[\alpha(x), y_{2}\right]\right\rangle \\
& -(-1)^{|x||y|}\left\langle\phi \otimes \psi,\left[\alpha(y), x_{1}\right] \otimes \alpha\left(x_{2}\right)\right\rangle-(-1)^{|x||y|}(-1)^{|y|\left|x_{1}\right|}\left\langle\phi \otimes \psi, \alpha\left(x_{1}\right) \otimes\left[\alpha(y), x_{2}\right]\right\rangle \\
& =\left\langle\Delta(\phi) \otimes \psi, \alpha(x) \otimes y_{1} \otimes \alpha\left(y_{2}\right)\right\rangle+(-1)^{|x|\left|y_{1}\right|}\left\langle\phi \otimes \Delta(\psi), \alpha\left(y_{1}\right) \otimes \alpha(x) \otimes y_{2}\right\rangle \\
& -(-1)^{|x||y|}\left\langle\Delta(\phi) \otimes \psi, \alpha(y) \otimes x_{1} \otimes \alpha\left(x_{2}\right)\right\rangle \\
& -(-1)^{|x||y|}(-1)^{|y|\left|x_{1}\right|}\left\langle\phi \otimes \Delta(\psi), \alpha\left(x_{1}\right) \otimes \alpha(y) \otimes x_{2}\right\rangle \\
& =\left\langle\phi_{1} \otimes \phi_{2} \otimes \psi, \alpha(x) \otimes y_{1} \otimes \alpha\left(y_{2}\right)\right\rangle+(-1)^{|x|\left|y_{1}\right|}\left\langle\phi \otimes \psi_{1} \otimes \psi_{2}, \alpha\left(y_{1}\right) \otimes \alpha(x) \otimes y_{2}\right\rangle \\
& -(-1)^{|x||y|}\left\langle\phi_{1} \otimes \phi_{2} \otimes \psi, \alpha(y) \otimes x_{1} \otimes \alpha\left(x_{2}\right)\right\rangle \\
& -(-1)^{|x||y|}(-1)^{|y|\left|x_{1}\right|}\left\langle\phi \otimes \psi_{1} \otimes \psi_{2}, \alpha\left(x_{1}\right) \otimes \alpha(y) \otimes x_{2}\right\rangle \\
& =\left\langle\alpha\left(\phi_{1}\right) \otimes \phi_{2} \otimes \alpha(\psi), x \otimes y_{1} \otimes y_{2}\right\rangle+(-1)^{|x|\left|y_{1}\right|}\left\langle\alpha(\phi) \otimes \alpha\left(\psi_{1}\right) \otimes \psi_{2}, y_{1} \otimes x \otimes y_{2}\right\rangle \\
& -(-1)^{|x||y|}\left\langle\alpha\left(\phi_{1}\right) \otimes \phi_{2} \otimes \alpha(\psi), y \otimes x_{1} \otimes x_{2}\right\rangle \\
& -(-1)^{|x||y|}(-1)^{|y|\left|x_{1}\right|}\left\langle\alpha(\phi) \otimes \alpha\left(\psi_{1}\right) \otimes \psi_{2}, x_{1} \otimes y \otimes x_{2}\right\rangle .
\end{aligned}
$$


Using, in addition, the skew-supersymmetric of the bracket and the cobracket in $\mathfrak{L}^{*}$, $\left(\left[\phi_{1}, \alpha(\psi)\right]=-(-1)^{\left|\phi_{1}\right||\psi|}\left[\alpha(\psi), \phi_{1}\right]\right.$ and $\left.\phi_{1} \otimes \phi_{2}=-(-1)^{\left|\phi_{1}\right|\left|\phi_{2}\right|} \phi_{2} \otimes \phi_{1}\right)$.

The above four terms become:

$$
\begin{aligned}
& =-(-1)^{|\phi||\psi|}(-1)^{|\psi|\left|\phi_{1}\right|}\left\langle\alpha\left(\phi_{1}\right) \otimes\left[\alpha(\psi), \phi_{2}\right], x \otimes y\right\rangle+(-1)^{|\phi|\left|\psi_{1}\right|}\left\langle\alpha\left(\psi_{1}\right) \otimes\left[\alpha(\phi), \psi_{2}\right], x \otimes y\right\rangle \\
& -(-1)^{|\phi||\psi|}\left\langle\left[\alpha(\psi), \phi_{1}\right] \otimes \alpha\left(\phi_{2}\right), x \otimes y\right\rangle+\left\langle\left[\alpha(\phi), \psi_{1}\right] \otimes \alpha\left(\psi_{2}\right), x \otimes y\right\rangle . \\
& =\left\langle a d_{\alpha(\phi)}(\Delta(\psi))-(-1)^{|\phi||\psi|} a d_{\alpha(\psi)}(\Delta(\phi)), x \otimes y\right\rangle
\end{aligned}
$$

This is exactly the right-hand side of (1.13).

\section{Matched pairs, Hom-Lie superbialgebras and Manin supertriples}

In this section, we introduce the notions of matched pair of Hom-Lie superalgebras and a Manin supertriple of Hom-Lie superalgebras. First, we recall the basics about representations of a Hom-Lie superalgebras.

Definition 2.1. ([2, 22, 223) Let $(\mathfrak{L},[\cdot, \cdot], \alpha)$ be a Hom-Lie superalgebra and $M=M_{\overline{0}} \oplus M_{\overline{1}}$ an arbitrary vector superspace. A representation of the Hom-Lie superalgebra with respect to $A \in \mathfrak{g l}(M)$, is an even linear map $\rho: \mathfrak{L} \rightarrow \operatorname{End}(M)$, such that $\rho\left(\mathfrak{L}_{\mathfrak{i}}\right)\left(M_{j}\right) \subset M_{i+j}$ where $i, j \in \mathbb{Z}_{2}$, and satisfying

$$
\rho(\alpha(x)) \circ A=A \circ \rho(x)
$$

and

$$
\rho([x, y]) \circ A=\rho(\alpha(x)) \circ \rho(y)-(-1)^{|x||y|} \rho(\alpha(y)) \circ \rho(x) .
$$

for all homogeneous elements $x, y \in \mathfrak{L}$.

We denote a representation by $(M, \rho, A)$. It is straightforward to see that $(\mathfrak{L}, a d, \alpha)$ is a representation, called the adjoint representation, see [4, 22.

Given a representation $(M, \rho, A)$, define $\rho^{*}: \mathfrak{L} \rightarrow \operatorname{End}\left(M^{*}\right)$ by

$$
\left\langle\rho^{*}(x)(\xi), \nu\right\rangle=-(-1)^{|x||\xi|}\langle\xi, \rho(x)(\nu)\rangle,
$$

$\forall x \in \mathfrak{L}, \xi \in M^{*}, \nu \in M$. This representation is called admissible representation with respect to $(M, \rho, A)$.

Now, let $\left(\mathfrak{g},[\cdot, \cdot]_{\mathfrak{g}}, \alpha_{\mathfrak{g}}\right)$ and $\left(\mathfrak{g}^{\prime},[\cdot, \cdot]_{\mathfrak{g}^{\prime}}, \alpha_{\mathfrak{g}^{\prime}}\right)$ be two multiplicative Hom-Lie superalgebras. Set $\mathfrak{g}=\mathfrak{g}_{\overline{0}} \oplus \mathfrak{g}_{\overline{1}}$

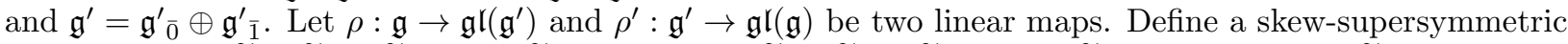
bracket $[\cdot, \cdot]_{\widetilde{G}}: \widetilde{G} \times \widetilde{G} \rightarrow \widetilde{G}$, where $\widetilde{G}$ is given by : $\widetilde{G}=\widetilde{G}_{\overline{0}} \oplus \widetilde{G}_{\overline{1}}$, where $\widetilde{G}_{\overline{0}}=\mathfrak{g}_{\overline{0}} \oplus \mathfrak{g}_{\overline{0}}^{\prime}$ and $\widetilde{G}_{\overline{1}}=\mathfrak{g}_{\overline{1}} \oplus \mathfrak{g}^{\prime}{ }_{\overline{1}}$. We set $\left|\left(x, x^{\prime}\right)\right|=|x|=\left|x^{\prime}\right|$ and $\left|\left(y, y^{\prime}\right)\right|=|y|=\left|y^{\prime}\right|$, for all homogeneous elements $x, y$ in $\mathfrak{g}$ and $x^{\prime}, y^{\prime}$ in $\mathfrak{g}^{\prime}$. Define $\widetilde{\alpha}: \widetilde{G} \rightarrow \widetilde{G}$ by

$$
\widetilde{\alpha}\left(x, x^{\prime}\right)=\left(\alpha_{\mathfrak{g}}(x), \alpha_{\mathfrak{g}^{\prime}}\left(x^{\prime}\right)\right),
$$

and the bracket $\widetilde{G}$ by

$$
\left[\left(x, x^{\prime}\right),\left(y, y^{\prime}\right)\right]_{\widetilde{G}}=\left([x, y]_{\mathfrak{g}}-(-1)^{|x||y|} \rho^{\prime}\left(y^{\prime}\right)(x)+\rho^{\prime}\left(x^{\prime}\right)(y),\left[x^{\prime}, y^{\prime}\right]_{\mathfrak{g}^{\prime}}+\rho(x)\left(y^{\prime}\right)-(-1)^{|x||y|} \rho(y)\left(x^{\prime}\right)\right) .
$$

Theorem 2.2. The triple $\left(\widetilde{G}=\mathfrak{g} \oplus \mathfrak{g}^{\prime},[\cdot, \cdot] \widetilde{G}, \widetilde{\alpha}\right)$, where $\widetilde{G},[\cdot, \cdot]_{\widetilde{G}}, \widetilde{\alpha}$ are defined above is a multiplicative Hom-Lie superalgebra if and only if $\rho$ and $\rho^{\prime}$ are representations of $\mathfrak{g}$ and $\mathfrak{g}^{\prime}$ respectively and the following conditions are satisfied

$$
\begin{aligned}
\rho\left(\alpha_{\mathfrak{g}}(z)\right)\left(\left[x^{\prime}, y^{\prime}\right]_{\mathfrak{g}^{\prime}}\right) & =\left[\rho(z)\left(x^{\prime}\right), \alpha_{\mathfrak{g}^{\prime}}\left(y^{\prime}\right)\right]_{\mathfrak{g}^{\prime}}+(-1)^{|x||z|}\left[\alpha_{\mathfrak{g}^{\prime}}\left(x^{\prime}\right), \rho(z)\left(y^{\prime}\right)\right]_{\mathfrak{g}^{\prime}} \\
& +(-1)^{|x||y|+|y||z|} \rho\left(\rho^{\prime}\left(y^{\prime}\right)(z)\right)\left(\alpha_{\mathfrak{g}^{\prime}}\left(x^{\prime}\right)\right)-(-1)^{|x||z|} \rho\left(\rho^{\prime}\left(x^{\prime}\right)(z)\right)\left(\alpha_{\mathfrak{g}^{\prime}}\left(y^{\prime}\right)\right) . \\
\rho^{\prime}\left(\alpha_{\mathfrak{g}^{\prime}}\left(z^{\prime}\right)\right)\left([x, y]_{\mathfrak{g}}\right) & =\left[\rho^{\prime}\left(z^{\prime}\right)(x), \alpha_{\mathfrak{g}}(y)\right]_{\mathfrak{g}}+(-1)^{|x||z|}\left[\alpha_{\mathfrak{g}}(x), \rho^{\prime}\left(z^{\prime}\right)(y)\right]_{\mathfrak{g}} \\
& +(-1)^{|x||y|+|y||z|} \rho^{\prime}\left(\rho(y)\left(z^{\prime}\right)\right)\left(\alpha_{\mathfrak{g}}(x)\right)-(-1)^{|x| z \mid} \rho^{\prime}\left(\rho(x)\left(z^{\prime}\right)\right)\left(\alpha_{\mathfrak{g}}(y)\right) .
\end{aligned}
$$


Proof. Assume $\left(\widetilde{G}=\mathfrak{g} \oplus \mathfrak{g}^{\prime},[\cdot, \cdot]_{\widetilde{G}}, \widetilde{\alpha}\right)$ is a multiplicative Hom-Lie superalgebra. The multiplicativity condition writes

$$
\widetilde{\alpha}\left(\left[\left(x, x^{\prime}\right),\left(y, y^{\prime}\right)\right]_{\widetilde{G}}\right)=\left[\widetilde{\alpha}\left(x, x^{\prime}\right), \widetilde{\alpha}\left(y, y^{\prime}\right)\right]_{\widetilde{G}} .
$$

Developing (2.7), leads to the first condition (2.1). Indeed

$$
\begin{array}{r}
\widetilde{\alpha}\left(\left[\left(x, x^{\prime}\right),\left(y, y^{\prime}\right)\right]_{\widetilde{G}}\right)=\left(\alpha_{\mathfrak{g}}\left([x, y]_{\mathfrak{g}}\right)-(-1)^{|x||y|} \alpha_{\mathfrak{g}}\left(\rho^{\prime}\left(y^{\prime}\right)(x)\right)+\alpha_{\mathfrak{g}}\left(\rho^{\prime}\left(x^{\prime}\right)(y)\right),\right. \\
\left.\alpha_{\mathfrak{g}^{\prime}}\left(\left[x^{\prime}, y^{\prime}\right]_{\mathfrak{g}^{\prime}}\right)+\alpha_{\mathfrak{g}^{\prime}}\left(\rho(x)\left(y^{\prime}\right)\right)-(-1)^{|x| y \mid} \alpha_{\mathfrak{g}^{\prime}}\left(\rho(y)\left(x^{\prime}\right)\right)\right) . \\
{\left[\widetilde{\alpha}\left(x, x^{\prime}\right), \widetilde{\alpha}\left(y, y^{\prime}\right)\right]_{\widetilde{G}}=\left(\left[\alpha_{\mathfrak{g}}(x), \alpha_{\mathfrak{g}}(y)\right]_{\mathfrak{g}}-(-1)^{|x||y|} \rho^{\prime}\left(\alpha_{\mathfrak{g}^{\prime}}\left(y^{\prime}\right)\right)\left(\alpha_{\mathfrak{g}}(x)\right)+\rho^{\prime}\left(\alpha_{\mathfrak{g}^{\prime}}\left(x^{\prime}\right)\right)\left(\alpha_{\mathfrak{g}}(y)\right),\right.} \\
\left.\left[\alpha_{\mathfrak{g}^{\prime}}\left(x^{\prime}\right), \alpha_{\mathfrak{g}^{\prime}}\left(y^{\prime}\right)\right]_{\mathfrak{g}^{\prime}}+\rho\left(\alpha_{\mathfrak{g}}(x)\right)\left(\alpha_{\mathfrak{g}^{\prime}}\left(y^{\prime}\right)\right)-(-1)^{|x||y|} \rho\left(\alpha_{\mathfrak{g}}(y)\right)\left(\alpha_{\mathfrak{g}^{\prime}}\left(x^{\prime}\right)\right)\right) .
\end{array}
$$

Since $\alpha_{\mathfrak{g}}$ and $\alpha_{\mathfrak{g}^{\prime}}$ are multiplicative, which implies that

$$
\begin{gathered}
\rho\left(\alpha_{\mathfrak{g}}(x)\right) \circ \alpha_{\mathfrak{g}^{\prime}}=\alpha_{\mathfrak{g}^{\prime}} \circ \rho(x), \\
\rho^{\prime}\left(\alpha_{\mathfrak{g}^{\prime}}\left(x^{\prime}\right)\right) \circ \alpha_{\mathfrak{g}}=\alpha_{\mathfrak{g}} \circ \rho^{\prime}\left(x^{\prime}\right) .
\end{gathered}
$$

Developing the Hom-super-Jacobi identity (1.2), that for $x, y, z \in \mathfrak{g}$ and $x^{\prime}, y^{\prime}, z^{\prime} \in \mathfrak{g}^{\prime}$,

$$
\begin{gathered}
(-1)^{\left|\left(x, x^{\prime}\right)\right|\left|\left(z, z^{\prime}\right)\right|}\left[\widetilde{\alpha}\left(x, x^{\prime}\right),\left[\left(y, y^{\prime}\right),\left(z, z^{\prime}\right)\right]_{\widetilde{G}}\right]_{\widetilde{G}}+(-1)^{\left|\left(y, y^{\prime}\right)\right|\left|\left(x, x^{\prime}\right)\right|}\left[\widetilde{\alpha}\left(y, y^{\prime}\right),\left[\left(z, z^{\prime}\right),\left(x, x^{\prime}\right)\right]_{\widetilde{G}}\right]_{\widetilde{G}} \\
+(-1)^{\left|\left(z, z^{\prime}\right)\right|\left|\left(y, y^{\prime}\right)\right|}\left[\widetilde{\alpha}\left(z, z^{\prime}\right),\left[\left(x, x^{\prime}\right),\left(y, y^{\prime}\right)\right]_{\widetilde{G}}\right]_{\widetilde{G}}=0,
\end{gathered}
$$

leads to the second condition (2.2). Indeed,

$$
\begin{aligned}
- & (-1)^{\left|\left(x, x^{\prime}\right)\right|\left|\left(z, z^{\prime}\right)\right|}\left[\widetilde{\alpha}\left(x, x^{\prime}\right),\left[\left(y, y^{\prime}\right),\left(z, z^{\prime}\right)\right]_{\widetilde{G}}\right]_{\widetilde{G}}=(-1)^{|x||z|}\left(\left[\alpha_{\mathfrak{g}}(x),[y, z]_{\mathfrak{g}}\right]_{\mathfrak{g}}-(-1)^{|y||z|}\left[\alpha_{\mathfrak{g}}(x), \rho^{\prime}\left(z^{\prime}\right)(y)\right]_{\mathfrak{g}}\right. \\
& +\left[\alpha_{\mathfrak{g}}(x), \rho^{\prime}\left(y^{\prime}\right)(z)\right]_{\mathfrak{g}}-(-1)^{|x|(|y|+|z|)} \rho^{\prime}\left(\left[y^{\prime}, z^{\prime}\right]_{\mathfrak{g}^{\prime}}\right)\left(\alpha_{\mathfrak{g}}(x)\right)-(-1)^{|x|(|y|+|z|)} \rho^{\prime}\left(\rho(y)\left(z^{\prime}\right)\right)\left(\alpha_{\mathfrak{g}}(x)\right) \\
& +(-1)^{|x|(|y|+|z|)}(-1)^{|y||z|} \rho^{\prime}\left(\rho(z)\left(y^{\prime}\right)\right)\left(\alpha_{\mathfrak{g}}(x)\right)+\rho^{\prime}\left(\alpha_{\mathfrak{g}^{\prime}}\left(x^{\prime}\right)\right)\left([y, z]_{\mathfrak{g}}\right) \\
& -(-1)^{|y||z|} \rho^{\prime}\left(\alpha_{\mathfrak{g}^{\prime}}\left(x^{\prime}\right)\right)\left(\rho^{\prime}\left(z^{\prime}\right)(y)\right)+\rho^{\prime}\left(\alpha_{\mathfrak{g}^{\prime}}\left(x^{\prime}\right)\right)\left(\rho^{\prime}\left(y^{\prime}\right)(z)\right),\left[\alpha_{\mathfrak{g}^{\prime}}\left(x^{\prime}\right),\left[y^{\prime}, z^{\prime}\right]_{\mathfrak{g}^{\prime}}\right]_{\mathfrak{g}^{\prime}} \\
& +\left[\alpha_{\mathfrak{g}^{\prime}}\left(x^{\prime}\right), \rho(y)\left(z^{\prime}\right)\right]_{\mathfrak{g}^{\prime}}-(-1)^{|y||z|}\left[\alpha_{\mathfrak{g}^{\prime}}\left(x^{\prime}\right), \rho(z)\left(y^{\prime}\right)\right]_{\mathfrak{g}^{\prime}}+\rho\left(\alpha_{\mathfrak{g}}(x)\right)\left(\left[y^{\prime}, z^{\prime}\right]_{\mathfrak{g}^{\prime}}\right) \\
& +\rho\left(\alpha_{\mathfrak{g}}(x)\right)\left(\rho(y)\left(z^{\prime}\right)\right)-(-1)^{|y||z|} \rho\left(\alpha_{\mathfrak{g}}(x)\right)\left(\rho(z)\left(y^{\prime}\right)\right)-(-1)^{|x|(|y|+|z|)} \rho\left([y, z]_{\mathfrak{g}}\right)\left(\alpha_{\mathfrak{g}^{\prime}}\left(x^{\prime}\right)\right) \\
& \left.+(-1)^{|x|(|y|+|z|)}(-1)^{|y||z|} \rho\left(\rho^{\prime}\left(z^{\prime}\right)(y)\right)\left(\alpha_{\mathfrak{g}^{\prime}}\left(x^{\prime}\right)\right)-(-1)^{|x|(|y|+|z|)} \rho\left(\rho^{\prime}\left(y^{\prime}\right)(z)\right)\left(\alpha_{\mathfrak{g}^{\prime}}\left(x^{\prime}\right)\right)\right) .
\end{aligned}
$$

Similarly, we compute :

$$
(-1)^{\left|\left(y, y^{\prime}\right)\right|\left|\left(x, x^{\prime}\right)\right|}\left[\widetilde{\alpha}\left(y, y^{\prime}\right),\left[\left(z, z^{\prime}\right),\left(x, x^{\prime}\right)\right]_{\widetilde{G}}\right]_{\widetilde{G}} \text { and }(-1)^{\left|\left(z, z^{\prime}\right)\right|\left|\left(y, y^{\prime}\right)\right|}\left[\widetilde{\alpha}\left(z, z^{\prime}\right),\left[\left(x, x^{\prime}\right),\left(y, y^{\prime}\right)\right]_{\widetilde{G}}\right]_{\widetilde{G}} .
$$

Setting $\left(|x|=\left|x^{\prime}\right|,|y|=\left|y^{\prime}\right|\right.$, and $\left.|z|=\left|z^{\prime}\right|\right)$ give

$$
\begin{gathered}
\rho\left([x, y]_{\mathfrak{g}}\right) \circ \alpha_{\mathfrak{g}^{\prime}}=\rho\left(\alpha_{\mathfrak{g}}(x)\right) \circ \rho(y)-(-1)^{|x||y|} \rho\left(\alpha_{\mathfrak{g}}(y)\right) \circ \rho(x), \\
\rho^{\prime}\left(\left[x^{\prime}, y^{\prime}\right]_{\mathfrak{g}^{\prime}}\right) \circ \alpha_{\mathfrak{g}}=\rho^{\prime}\left(\alpha_{\mathfrak{g}^{\prime}}\left(x^{\prime}\right)\right) \circ \rho^{\prime}\left(y^{\prime}\right)-(-1)^{\left|x^{\prime}\right|\left|y^{\prime}\right|} \rho^{\prime}\left(\alpha_{\mathfrak{g}^{\prime}}\left(y^{\prime}\right)\right) \circ \rho^{\prime}\left(x^{\prime}\right),
\end{gathered}
$$

which implies (2.5), and (2.6).

By Eqs. (2.8) and (2.10), we deduce that $\rho$ is a representation of the Hom-Lie superalgebra $\left(\mathfrak{g},[\cdot, \cdot]_{\mathfrak{g}}, \alpha_{\mathfrak{g}}\right)$ on $\mathfrak{g}^{\prime}$ with respect to $\alpha_{\mathfrak{g}^{\prime}}$. By Eqs. (2.9) and (2.11), we deduce that $\rho^{\prime}$ is a representation of the Hom-Lie superalgebra $\left(\mathfrak{g}^{\prime},[\cdot, \cdot]_{\mathfrak{g}^{\prime}}, \alpha_{\mathfrak{g}^{\prime}}\right)$ on $\mathfrak{g}$ with respect to $\alpha_{\mathfrak{g}}$.

Example 2.3 (See 2]). Given a representation $\left(V,[.,]_{V}, \beta\right)$ of a Hom-Lie superalgebra $(G,[\cdot, \cdot], \alpha)$. Set $\widetilde{G}=G \oplus V$ and $\widetilde{G_{k}}=G_{k} \oplus V_{k}$. If $x \in G_{i}$ and $v \in V_{i}\left(i \in \mathbb{Z}_{2}\right)$, we denote $|(x, v)|=|x|$.

Define a skew-supersymmetric bracket $[\cdot, \cdot]_{\widetilde{G}}: \wedge^{2}(G \oplus V) \rightarrow G \oplus V$ by

$$
[(x, \mu),(y, v)]_{\widetilde{G}}=\left([x, y],[x, v]_{V}-(-1)^{|x||y|}[y, \mu]_{V}\right) .
$$

Define $\widetilde{\alpha}: G \oplus V \rightarrow G \oplus V$ by $\widetilde{\alpha}(x, v)=(\alpha(x), \beta(v))$. Then $\left(G \oplus V,[-,-]_{\widetilde{G}}, \widetilde{\alpha}\right)$ is a Hom-Lie superalgebra, which we call the semi-direct product of the Hom-Lie superalgebra $(G,[\cdot, \cdot], \alpha)$ by $V$. 
Definition 2.4. For any $x \in \mathfrak{g}$, define $a d_{x}: \wedge^{n} \mathfrak{g} \rightarrow \wedge^{n} \mathfrak{g}$ by $a d_{x} P=[x, P]_{\mathfrak{g}}$. Its dual map $a d^{*}: \wedge^{n} \mathfrak{g}^{*} \rightarrow \wedge^{n} \mathfrak{g}^{*}$ is defined as

$$
\left\langle a d_{x}(y), \xi\right\rangle=-(-1)^{|x||y|}\left\langle y, a d_{x}^{*}(\xi)\right\rangle
$$

for all $x, y \in \mathfrak{g}$, and $\xi \in \mathfrak{g}^{*}$, where the pairing $\langle$,$\rangle is supersymmetric, i.e. \langle x, \xi\rangle=(-1)^{|x||\xi|}\langle\xi, x\rangle$, for $x \in \mathfrak{g}$, and $\xi \in \mathfrak{g}^{*}$. More precisely, for any $\xi_{1}, \cdots, \xi_{n} \in \mathfrak{g}^{*}$, we have

$$
a d_{x}^{*}\left(\xi_{1} \wedge \cdots \wedge \xi_{n}\right)=\sum_{i=1}^{n}(-1)^{|x|\left(\left|\xi_{1}\right|+\left|\xi_{2}\right|+\cdots+\left|\xi_{i-1}\right|\right)} \alpha_{\mathfrak{g}}^{*}\left(\xi_{1}\right) \wedge \cdots \wedge a d_{x}^{*}\left(\xi_{i}\right) \wedge \cdots \wedge \alpha_{\mathfrak{g}}^{*}\left(\xi_{n}\right) .
$$

Definition 2.5. A matched pairs of Hom-Lie superalgebras, which we denote by $\left(\mathfrak{g}, \mathfrak{g}^{\prime}, \rho, \rho^{\prime}\right)$, consists of two Hom-Lie superalgebras $\left(\mathfrak{g},[\cdot, \cdot]_{\mathfrak{g}}, \alpha_{\mathfrak{g}}\right)$ and $\left(\mathfrak{g}^{\prime},[\cdot, \cdot]_{\mathfrak{g}^{\prime}}, \alpha_{\mathfrak{g}^{\prime}}\right)$, together with representations $\rho: \mathfrak{g} \rightarrow \mathfrak{g l}\left(\mathfrak{g}^{\prime}\right)$ and $\rho^{\prime}: \mathfrak{g}^{\prime} \rightarrow \mathfrak{g l}(\mathfrak{g})$ with respect to $\alpha_{\mathfrak{g}^{\prime}}$ and $\alpha_{\mathfrak{g}}$ respectively, such that the compatibility conditions (2.5) and (2.6) are satisfied.

In the following, we concentrate on the case that $\mathfrak{g}^{\prime}$ is $\mathfrak{g}^{*}$, the dual space of $\mathfrak{g}$, and $\alpha_{\mathfrak{g}^{\prime}}=\alpha_{\mathfrak{g}}^{*}, \rho=a d^{*}$, $\rho^{\prime}=\mathfrak{a d}^{*}$, where $\mathfrak{a d}^{*}$ is the dual map of $\mathfrak{a} \mathfrak{d}$. Notice that $a d$ and $\mathfrak{a} \mathfrak{d}$ are the adjoint representations associated to Hom-Lie superalgebras $\mathfrak{g}$ and $\mathfrak{g}^{*}$ respectively. Let $x, y, z$ be elements in $\mathfrak{g}$ and $\xi, \eta$ elements in $\mathfrak{g}^{*}$.

For a Hom-Lie superalgebra $\left(\mathfrak{g},[\cdot, \cdot]_{\mathfrak{g}}, \alpha_{\mathfrak{g}}\right)$ (resp. $\left.\left(\mathfrak{g}^{*},[\cdot, \cdot]_{\mathfrak{g}^{*}}, \alpha_{\mathfrak{g}^{*}}\right)\right)$, let $\Delta^{*}: \mathfrak{g}^{*} \rightarrow \wedge^{2} \mathfrak{g}^{*}\left(\operatorname{resp} . \Delta: \mathfrak{g} \rightarrow \wedge^{2} \mathfrak{g}\right)$ be the dual map of $[\cdot, \cdot]_{\mathfrak{g}}: \wedge^{2} \mathfrak{g} \rightarrow \mathfrak{g}$ (resp. $\left.[\cdot, \cdot]_{\mathfrak{g}^{*}}: \wedge^{2} \mathfrak{g}^{*} \rightarrow \mathfrak{g}^{*}\right)$, i.e.

$$
\left\langle\Delta^{*}(\xi), x \wedge y\right\rangle=\left\langle\xi,[x, y]_{\mathfrak{g}}\right\rangle, \quad\langle\Delta(x), \xi \wedge \eta\rangle=\left\langle x,[\xi, \eta]_{\mathfrak{g}^{*}}\right\rangle .
$$

A Hom-Lie superalgebra $(\mathfrak{L},[\cdot, \cdot], \alpha)$ is called admissible if its adjoint representation is admissible, that is $\left[\left(I d-\alpha^{2}\right)(x), \alpha(y)\right]=0$. In particular, if a Hom-Lie superalgebra $(\mathfrak{L},[\cdot, \cdot], \alpha)$ is involutive, that is $\alpha^{2}=I d$, then it is admissible.

Proposition 2.6. A pair of admissible Hom-Lie superalgebras $\left(\mathfrak{g},[\cdot, \cdot]_{\mathfrak{g}}, \alpha_{\mathfrak{g}}\right)$ and $\left(\mathfrak{g}^{*},[\cdot, \cdot]_{\mathfrak{g}^{*}}, \alpha_{\mathfrak{g}}^{*}\right)$ determines a Hom-Lie superbialgebra $\left(\mathfrak{g},[\cdot, \cdot]_{\mathfrak{g}}, \Delta, \alpha_{\mathfrak{g}}\right)$, where $\Delta$ is the dual operation of $[\cdot, \cdot]_{\mathfrak{g}^{*}}$ if

$$
\begin{aligned}
\left\langle\Delta\left([x, y]_{\mathfrak{g}}\right), \alpha_{\mathfrak{g}}^{*}(\xi) \wedge \eta\right\rangle & =\left\langle a d_{\alpha_{\mathfrak{g}}(x)}(\Delta(y)), \alpha_{\mathfrak{g}}^{*}(\xi) \wedge \eta\right\rangle-(-1)^{|x||y|}\left\langle a d_{\alpha_{\mathfrak{g}}(y)}(\Delta(x)), \alpha_{\mathfrak{g}}^{*}(\xi) \wedge \eta\right\rangle, \\
\left\langle\Delta^{*}\left([\xi, \eta]_{\mathfrak{g}^{*}}\right), \alpha_{\mathfrak{g}}(x) \wedge y\right\rangle & =\left\langle\mathfrak{a} \mathfrak{d}_{\alpha_{\mathfrak{g}}^{*}(\xi)}\left(\Delta^{*}(\eta)\right), \alpha_{\mathfrak{g}}(x) \wedge y\right\rangle-(-1)^{|\xi||\eta|}\left\langle\mathfrak{a} \mathfrak{d}_{\alpha_{\mathfrak{g}}^{*}(\eta)}\left(\Delta^{*}(\xi)\right), \alpha_{\mathfrak{g}}(x) \wedge y\right\rangle .
\end{aligned}
$$

Remark 2.7. Following Bai and Sheng, we may denote this Hom-Lie superbialgebra by $\left(\mathfrak{g}, \mathfrak{g}^{*}\right)$.

Theorem 2.8. Let $\left(\mathfrak{g},[\cdot, \cdot]_{\mathfrak{g}}, \alpha_{\mathfrak{g}}\right)$ and $\left(\mathfrak{g}^{*},[\cdot, \cdot]_{\mathfrak{g}^{*}}, \alpha_{\mathfrak{g}}^{*}\right)$ be a pair of admissible Hom-Lie superalgebra. Then $\left(\mathfrak{g},[\cdot, \cdot]_{\mathfrak{g}}, \Delta, \alpha_{\mathfrak{g}}\right)$, where $\Delta$ is the dual of $[\cdot, \cdot]_{\mathfrak{g}^{*}}$, is a Hom-Lie superbialgebra if and only if $\left(\mathfrak{g},[\cdot, \cdot]_{\mathfrak{g}}, \alpha_{\mathfrak{g}}\right)$ and $\left(\mathfrak{g}^{*},[\cdot, \cdot]_{\mathfrak{g}^{*}}, \alpha_{\mathfrak{g}}^{*}\right)$ is a matched pairs of Hom-Lie superalgebras, i.e. $\left(\mathfrak{g} \oplus \mathfrak{g}^{*},[\cdot, \cdot]_{\widetilde{G}}, \alpha_{\mathfrak{g}} \oplus \alpha_{\mathfrak{g}}^{*}\right)$ is a multiplicative Hom-Lie superalgebra, where $[\cdot, \cdot]_{\widetilde{G}}$ is given by Eq. 2.4), in which $\rho=a d^{*}$ and $\rho^{\prime}=\mathfrak{a d}^{*}$.

Proof. By Theorem 2.2, two admissible Hom-Lie superalgebras $\left(\mathfrak{g},[\cdot, \cdot]_{\mathfrak{g}}, \alpha_{\mathfrak{g}}\right)$ and $\left(\mathfrak{g}^{*},[\cdot, \cdot]_{\mathfrak{g}^{*}}, \alpha_{\mathfrak{g}}^{*}\right)$ form a matched pair of Hom-Lie superalgebras if and only if

$$
\begin{aligned}
a d_{\alpha_{\mathfrak{g}}(z)}^{*}\left([\xi, \eta]_{\mathfrak{g}^{*}}\right)= & {\left[a d_{z}^{*}(\xi), \alpha_{\mathfrak{g}}^{*}(\eta)\right]_{\mathfrak{g}^{*}}+(-1)^{|\xi||z|}\left[\alpha_{\mathfrak{g}}^{*}(\xi), a d_{z}^{*}(\eta)\right]_{\mathfrak{g}^{*}} } \\
& +(-1)^{|\xi||\eta|+|\eta||z|} a d_{\mathfrak{a d}_{\eta}^{*}(z)}^{*}\left(\alpha_{\mathfrak{g}}^{*}(\xi)\right)-(-1)^{|\xi||z|} a d_{\mathfrak{a d}_{\xi}^{*}(z)}^{*}\left(\alpha_{\mathfrak{g}}^{*}(\eta)\right) . \\
\mathfrak{a d}_{\alpha_{\mathfrak{g}}^{*}(\xi)}\left([x, y]_{\mathfrak{g}}\right)= & {\left[\mathfrak{a} \mathfrak{d}_{\xi}^{*}(x), \alpha_{\mathfrak{g}}(y)\right]_{\mathfrak{g}}+(-1)^{|x||\xi|}\left[\alpha_{\mathfrak{g}}(x), \mathfrak{a} \mathfrak{d}_{\xi}^{*}(y)\right]_{\mathfrak{g}} } \\
& +(-1)^{|x||y|+|y||\xi|} \mathfrak{a d}_{a d_{y}^{*}(\xi)}\left(\alpha_{\mathfrak{g}}(x)\right)-(-1)^{|x||\xi|} \mathfrak{a d}_{a d_{x}^{*}(\xi)}^{*}\left(\alpha_{\mathfrak{g}}(y)\right) .
\end{aligned}
$$

Since $\left(\mathfrak{g},[\cdot, \cdot]_{\mathfrak{g}}, \alpha_{\mathfrak{g}}\right)$ and $\left(\mathfrak{g}^{*},[\cdot, \cdot]_{\mathfrak{g}^{*}}, \alpha_{\mathfrak{g}}^{*}\right)$ are admissible Hom-Lie superalgebras i.e. $\left(\alpha_{\mathfrak{g}}^{2}=I d\right.$, and $\left.\left(\alpha_{\mathfrak{g}}^{*}\right)^{2}=I d\right)$, see Lemma 2.9 in [23]. By Eq. (2.17), we get

$$
\begin{aligned}
0 & =\left\langle-\mathfrak{a} \mathfrak{d}_{\alpha_{\mathfrak{g}}^{*}(\xi)}^{*}\left([x, y]_{\mathfrak{g}}\right)+\left[\mathfrak{a} \mathfrak{d}_{\xi}^{*}(x), \alpha_{\mathfrak{g}}(y)\right]_{\mathfrak{g}}+(-1)^{|x||\xi|}\left[\alpha_{\mathfrak{g}}(x), \mathfrak{a} \mathfrak{d}_{\xi}^{*}(y)\right]_{\mathfrak{g}}+(-1)^{|x||y|+|y||\xi|} \mathfrak{a} \mathfrak{d}_{a d_{\mathfrak{y}}^{*}(\xi)}^{*}\left(\alpha_{\mathfrak{g}}(x)\right)\right. \\
& \left.-(-1)^{|x||\xi|} \mathfrak{a d}_{a d_{x}^{*}(\xi)}\left(\alpha_{\mathfrak{g}}(y)\right), \eta\right\rangle \\
& =(-1)^{|\xi|(|x|+|y|)}\left\langle[x, y]_{\mathfrak{g}},\left[\alpha_{\mathfrak{g}}^{*}(\xi), \eta\right]_{\mathfrak{g}^{*}}\right\rangle-(-1)^{|y|(|x|+|\xi|)}\left\langle a d_{\alpha_{\mathfrak{g}}(y)}\left(\mathfrak{a} \mathfrak{d}_{\xi}^{*}(x)\right), \eta\right\rangle+(-1)^{|x||\xi|}\left\langle a d_{\alpha_{\mathfrak{g}}(x)}\left(\mathfrak{a} \mathfrak{d}_{\xi}^{*}(y)\right), \eta\right\rangle \\
& -(-1)^{|\xi|(|x|+|y|)}\left\langle\alpha_{\mathfrak{g}}(x),\left[a d_{y}^{*}(\xi), \eta\right]_{\mathfrak{g}^{*}}\right\rangle+(-1)^{|x||\xi|}(-1)^{|y|(|x|+|\xi|)}\left\langle\alpha_{\mathfrak{g}}(y),\left[a d_{x}^{*}(\xi), \eta\right]_{\mathfrak{g}^{*}}\right\rangle \\
& =(-1)^{|\xi|(|x|+|y|)}\left\langle[x, y]_{\mathfrak{g}},\left[\alpha_{\mathfrak{g}}^{*}(\xi), \eta\right]_{\mathfrak{g}^{*}}\right\rangle-(-1)^{|x||\xi|}\left\langle x,\left[\xi, a d_{\alpha_{\mathfrak{g}}(y)}^{*}(\eta)\right]_{\mathfrak{g}^{*}}\right\rangle+(-1)^{|y|(|x|+|\xi|)}\left\langle y,\left[\xi, a d_{\alpha_{\mathfrak{g}}(x)}(\eta)\right]_{\mathfrak{g}^{*}}\right\rangle \\
& -(-1)^{|\xi|(|x|+|y|)}\left\langle x,\left[\alpha_{\mathfrak{g}}^{*}\left(a d_{y}^{*}(\xi)\right), \alpha_{\mathfrak{g}}^{*}(\eta)\right]_{\mathfrak{g}^{*}}\right\rangle+(-1)^{|x||\xi|}(-1)^{|y|(|x|+|\xi|)}\left\langle y,\left[\alpha_{\mathfrak{g}}^{*}\left(a d_{x}^{*}(\xi)\right), \alpha_{\mathfrak{g}}^{*}(\eta)\right]_{\mathfrak{g}^{*}}\right\rangle,
\end{aligned}
$$




$$
\begin{aligned}
& =(-1)^{|\xi|(|x|+|y|)}\left\langle[x, y]_{\mathfrak{g}},\left[\alpha_{\mathfrak{g}}^{*}(\xi), \eta\right]_{\mathfrak{g}^{*}}\right\rangle-(-1)^{|x||\xi|}\left\langle x,\left[\left(\alpha_{\mathfrak{g}}^{*}\right)^{2}(\xi), a d_{\alpha_{\mathfrak{g}}(y)}^{*}(\eta)\right]_{\mathfrak{g}^{*}}\right\rangle \\
& +(-1)^{|y|(|x|+|\xi|)}\left\langle y,\left[\left(\alpha_{\mathfrak{g}}^{*}\right)^{2}(\xi), a d_{\alpha_{\mathfrak{g}}(x)}^{*}(\eta)\right]_{\mathfrak{g}^{*}}\right\rangle-(-1)^{|\xi|(|x|+|y|)}\left\langle x,\left[a d_{\alpha_{\mathfrak{g}}(y)}^{*}\left(\alpha_{\mathfrak{g}}^{*}(\xi)\right), \alpha_{\mathfrak{g}}^{*}(\eta)\right]_{\mathfrak{g}^{*}}\right\rangle \\
& +(-1)^{|x||\xi|}(-1)^{|y|(|x|+|\xi|)}\left\langle y,\left[a d_{\alpha_{\mathfrak{g}}(x)}^{*}\left(\alpha_{\mathfrak{g}}^{*}(\xi)\right), \alpha_{\mathfrak{g}}^{*}(\eta)\right]_{\mathfrak{g}^{*}}\right\rangle \\
& =(-1)^{|\xi|(|x|+|y|)}\left\langle\Delta\left([x, y]_{\mathfrak{g}}\right), \alpha_{\mathfrak{g}}^{*}(\xi) \wedge \eta\right\rangle-(-1)^{|x||\xi|}\left\langle\Delta(x),\left(\alpha_{\mathfrak{g}}^{*}\right)^{2}(\xi) \wedge a d_{\alpha_{\mathfrak{g}}(y)}^{*}(\eta)\right\rangle \\
& +(-1)^{|y|(|x|+|\xi|)}\left\langle\Delta(y),\left(\alpha_{\mathfrak{g}}^{*}\right)^{2}(\xi) \wedge a d_{\alpha_{\mathfrak{g}}(x)}^{*}(\eta)\right\rangle-(-1)^{|\xi|(|x|+|y|)}\left\langle\Delta(x), a d_{\alpha_{\mathfrak{g}}(y)}^{*}\left(\alpha_{\mathfrak{g}}^{*}(\xi)\right) \wedge \alpha_{\mathfrak{g}}^{*}(\eta)\right\rangle \\
& +(-1)^{|x| \xi \mid}(-1)^{|y|(|x|+|\xi|)}\left\langle\Delta(y), a d_{\alpha_{\mathfrak{g}}(x)}^{*}\left(\alpha_{\mathfrak{g}}^{*}(\xi)\right) \wedge \alpha_{\mathfrak{g}}^{*}(\eta)\right\rangle,
\end{aligned}
$$

which implies that

$$
\begin{aligned}
\left\langle\Delta\left([x, y]_{\mathfrak{g}}\right), \alpha_{\mathfrak{g}}^{*}(\xi) \wedge \eta\right\rangle & =-(-1)^{|x||y|}\left\langle\Delta(y), a d_{\alpha_{\mathfrak{g}}(x)}^{*}\left(\alpha_{\mathfrak{g}}^{*}(\xi)\right) \wedge \alpha_{\mathfrak{g}}^{*}(\eta)+(-1)^{|x||\xi|}\left(\alpha_{\mathfrak{g}}^{*}\right)^{2}(\xi) \wedge a d_{\alpha_{\mathfrak{g}}(x)}^{*}(\eta)\right\rangle \\
& +\left\langle\Delta(x), a d_{\alpha_{\mathfrak{g}}(y)}^{*}\left(\alpha_{\mathfrak{g}}^{*}(\xi) \wedge \wedge \alpha_{\mathfrak{g}}^{*}(\eta)+(-1)^{|y||\xi|}\left(\alpha_{\mathfrak{g}}^{*}\right)^{2}(\xi) \wedge a d_{\alpha_{\mathfrak{g}}(y)}^{*}(\eta)\right\rangle\right. \\
& =-(-1)^{|x||y|}\left\langle\Delta(y), a d_{\alpha_{\mathfrak{g}}(x)}^{*}\left(\alpha_{\mathfrak{g}}^{*}(\xi) \wedge \eta\right)\right\rangle+\left\langle\Delta(x), a d_{\alpha_{\mathfrak{g}}(y)}^{*}\left(\alpha_{\mathfrak{g}}^{*}(\xi) \wedge \eta\right)\right\rangle \\
& =\left\langle a d_{\alpha_{\mathfrak{g}}(x)}(\Delta(y)), \alpha_{\mathfrak{g}}^{*}(\xi) \wedge \eta\right\rangle-(-1)^{|x||y|}\left\langle a d_{\alpha_{\mathfrak{g}}(y)}(\Delta(x)), \alpha_{\mathfrak{g}}^{*}(\xi) \wedge \eta\right\rangle,
\end{aligned}
$$

which is exactly Eq. (2.14). Similarly, one deduces that Eq. (2.16) is equivalent to Eq. (2.15).

Let $V$ be a superspace and $\langle\rangle:, V^{*} \times V \rightarrow \mathbb{K}$ be the canonical pairing. Then we identify $V$ with $V^{*}$ by the pairing $\langle x, \xi\rangle=(-1)^{|x||\xi|}\langle\xi, x\rangle, x \in V$ and $\xi \in V^{*}$. On the other hand, we shall say that a bilinear form $(\mid): V \times V \rightarrow \mathbb{K}$ is supersymmetric if $(v \mid \omega)=(-1)^{|v||\omega|}(\omega \mid v)$.

Definition 2.9. A Manin supertriple of Hom-Lie superalgebras is a triple of Hom-Lie superalgebras $\left(\mathfrak{M}, \mathfrak{g}, \mathfrak{g}^{\prime}\right)$ together with a nondegenerate supersymmetric bilinear form $S$ on $\mathfrak{M}$ such that

1. $S$ is invariant, i.e. for any $x, y, z \in \mathfrak{M}$, we have

$$
\begin{aligned}
S\left([x, y]_{\mathfrak{M}}, z\right) & =S\left(x,[y, z]_{\mathfrak{M}}\right), \\
S\left(\phi_{\mathfrak{M}}(x), y\right) & =S\left(x, \phi_{\mathfrak{M}}(y)\right) .
\end{aligned}
$$

2. $\mathfrak{g}$ and $\mathfrak{g}^{\prime}$ are isotropic Hom-Lie sub-superalgebra of $\mathfrak{M}$, such that $\mathfrak{M}=\mathfrak{g} \oplus \mathfrak{g}^{\prime}$ as vector superspace.

Proposition 2.10. Let $\left(\mathfrak{g}, \mathfrak{g}^{*}\right)$ be a Hom-Lie superbialgebra in the sense of Proposition [2.6. Then ( $\mathfrak{g} \oplus$ $\left.\mathfrak{g}^{*}, \mathfrak{g}, \mathfrak{g}^{*}\right)$ is a Manin supertriple of Hom-Lie superalgebras.

Proof. Let $\left(\mathfrak{g}, \mathfrak{g}^{*}\right)$ be a Hom-Lie superbialgebra in the sense of Proposition 2.6. i.e. $\mathfrak{g}$ and $\mathfrak{g}^{*}$ are admissible Hom-Lie superalgebras such that Eqs. (2.14), (2.15) are satisfied. By Theorem 2.8, we know that $(\mathfrak{g} \oplus$ $\left.\mathfrak{g}^{*},[\cdot, \cdot]_{\mathfrak{g} \oplus \mathfrak{g}^{*}}, \alpha_{\mathfrak{g}} \oplus \alpha_{\mathfrak{g}}^{*}\right)$ is a Hom-Lie superalgebra, where $[-,-]_{\mathfrak{g} \oplus \mathfrak{g}^{*}}$ is given by

$$
[x+\xi, y+\eta]_{\mathfrak{g} \oplus \mathfrak{g}^{*}}=[x, y]_{\mathfrak{g}}+[\xi, \eta]_{\mathfrak{g}^{*}}+a d_{x}^{*}(\eta)-(-1)^{|x||y|} a d_{y}^{*}(\xi)+\mathfrak{a} \mathfrak{d}_{\xi}^{*}(y)-(-1)^{|x||y|} \mathfrak{a} \mathfrak{d}_{\eta}^{*}(x),
$$

for all homogeneous elements $x, y$ and $\xi, \eta$ in $\mathfrak{g}$ and $\mathfrak{g}^{*}$ respectively. From the construction above we have $|x|=|\xi|$ and $|y|=|\eta|$.

Furthermore, there is an obvious supersymmetric bilinear form on $\mathfrak{g} \oplus \mathfrak{g}^{*}$ :

$$
S(x+\xi, y+\eta)=\langle x, \eta\rangle+\langle\xi, y\rangle=\langle x, \eta\rangle+(-1)^{|\xi||y|}\langle y, \xi\rangle .
$$

It's straightforward, using the supersymmetry and $\left\langle a d_{x}(y), \xi\right\rangle=-(-1)^{|x||y|}\left\langle y, a d_{x}^{*}(\xi)\right\rangle$, with $|x|=|\xi|$ and $|y|=|\eta|$, that Eqs. (2.18) and (2.19) are satisfied, i.e. the bilinear form defined by Eq. (2.21) is invariant.

Conversely, if $\left(\mathfrak{g} \oplus \mathfrak{g}^{*}, \mathfrak{g}, \mathfrak{g}^{*}\right)$ is a Manin supertriple of Hom-Lie superalgebras with the invariant bilinear from $S$ given by Eq. (2.21), then for any $x, y \in \mathfrak{g}$ and $\xi, \eta \in \mathfrak{g}^{*}$, we have the natural scalar product on $\mathfrak{g} \oplus \mathfrak{g}^{*}$ defined by

$$
S(x, y)=0, \quad S(\xi, \eta)=0, \quad S(x, \xi)=\langle x, \xi\rangle, \quad x, y \in \mathfrak{g}, \xi, \eta \in \mathfrak{g}^{*} .
$$

Due to the invariance of $S$, we have

$$
\begin{aligned}
S\left([x, \xi]_{\mathfrak{g} \oplus \mathfrak{g}^{*}}, y\right) & =(-1)^{|y|(|x|+|\xi|)} S\left(y,[x, \xi]_{\mathfrak{g} \oplus \mathfrak{g}^{*}}\right)=(-1)^{|y|(|x|+|\xi|)} S\left([y, x]_{\mathfrak{g} \oplus \mathfrak{g}^{*}}, \xi\right)=(-1)^{|y|(|x|+|\xi|)} S\left([y, x]_{\mathfrak{g}}, \xi\right) \\
& =-(-1)^{|y||\xi|}\left\langle a d_{x}(y), \xi\right\rangle=(-1)^{|y|(|x|+|\xi|)}\left\langle y, a d_{x}^{*}(\xi)\right\rangle=\left\langle a d_{x}^{*}(\xi), y\right\rangle,
\end{aligned}
$$


$S\left([x, \xi]_{\mathfrak{g} \oplus \mathfrak{g}^{*}}, \eta\right)=S\left(x,[\xi, \eta]_{\mathfrak{g} \oplus \mathfrak{g}^{*}}\right)=S\left(x,[\xi, \eta]_{\mathfrak{g}^{*}}\right)=\left\langle x, \mathfrak{a} \mathfrak{d}_{\xi}(\eta)\right\rangle=-(-1)^{|x||\xi|}\left\langle\mathfrak{a} \mathfrak{d}_{\xi}^{*}(x), \eta\right\rangle$, which implies that :

$$
[x, \xi]_{\mathfrak{g} \oplus \mathfrak{g}^{*}}=a d_{x}^{*}(\xi)-(-1)^{|x||\xi|} \mathfrak{a} \mathfrak{d}_{\xi}^{*}(x),
$$

that is, the Hom-Lie bracket on $\mathfrak{g} \oplus \mathfrak{g}^{*}$ is given by Eq. (2.20). Therefore, $\left(\mathfrak{g}, \mathfrak{g}^{*}, a d^{*}, \mathfrak{a d}{ }^{*}\right)$ is a matched pair of Hom-Lie superalgebras and hence $\left(\mathfrak{g}, \mathfrak{g}^{*}\right)$ is a Hom-Lie superbialgebra. Note that we deduce naturally that both $\mathfrak{g}$ and $\mathfrak{g}^{*}$ are admissible Hom-Lie superalgebras.

Summarizing the above study, Theorem 2.8 and Proposition 2.10, we have the following conclusion.

Theorem 2.11. Let $\left(\mathfrak{g},[\cdot, \cdot]_{\mathfrak{g}}, \alpha_{\mathfrak{g}}\right)$ and $\left(\mathfrak{g}^{*},[\cdot, \cdot]_{\mathfrak{g}^{*}}, \alpha_{\mathfrak{g}}^{*}\right)$ be two admissible Hom-Lie superalgebras. Then the following conditions are equivalent.

1. $\left(\mathfrak{g}, \mathfrak{g}^{*}\right)$ is a Hom-Lie superbialgebra in the sense of Proposition [2.6.

2. $\left(\mathfrak{g}, \mathfrak{g}^{*}, a d^{*}, \mathfrak{a} \mathfrak{d}^{*}\right)$ is a matched pair of Hom-Lie superalgebras.

3. $\left(\mathfrak{g} \oplus \mathfrak{g}^{*}, \mathfrak{g}, \mathfrak{g}^{*}\right)$ is a Manin supertriple of Hom-Lie superalgebras with the invariant bilinear from (2.21).

\section{Coboundary and quasi-triangular Hom-Lie superbialgebras}

In this section, we define and study coboundary Hom-Lie superbialgebras and quasi-triangular Hom-Lie superbialgebras. Then we show how a coboundary or a quasi-triangular Hom-Lie superbialgebra can be constructed from a Hom-Lie superalgebra and an $r$-matrix.

Definition 3.1. A (multiplicative) coboundary Hom-Lie superbialgebra $(\mathfrak{L},[\cdot, \cdot], \Delta, \alpha, r)$ consists of a (multiplicative) Hom-Lie superbialgebra $(\mathfrak{L},[\cdot, \cdot], \Delta, \alpha)$ and an element $r=\sum r_{1} \otimes r_{2} \in \mathfrak{L}^{\otimes 2}$ such that $\alpha^{\otimes 2}(r)=r$ and

$$
\Delta(x)=a d_{x}(r)=\sum\left[x, r_{1}\right] \otimes \alpha\left(r_{2}\right)+(-1)^{|x|\left|r_{1}\right|} \alpha\left(r_{1}\right) \otimes\left[x, r_{2}\right]
$$

for all $x \in \mathfrak{L}$.

Remark 3.2 .

$$
\Delta(x)=(-1)^{|x||r|}\left(\sum\left[x, r_{1}\right] \otimes \alpha\left(r_{2}\right)+(-1)^{|x|\left|r_{1}\right|} \alpha\left(r_{1}\right) \otimes\left[x, r_{2}\right]\right)
$$

for $x \in \mathfrak{L}$, where the parity $|r|$ of $r$ is defined as follows : since we assume $r$ is homogenous, there exists $|r| \in$ $\mathbb{Z}_{2}$, such that $r$ can be written as $r=\sum r_{1} \otimes r_{2} \in \mathfrak{L}^{\otimes 2}, r_{1}, r_{2}$ are homogenous elements with $|r|=\left|r_{1}\right|+\left|r_{2}\right|$. (Note that equation (3.2) and (1.3) show that we have $|r|=\overline{0}$, namely $\left|r_{1}\right|=\left|r_{2}\right|$ ). So we get (3.1).

Definition 3.3. The classical Yang-Baxter equation (CYBE):

$$
c(r)=\left[r_{12}, r_{13}\right]+\left[r_{12}, r_{23}\right]+\left[r_{13}, r_{23}\right]=0,
$$

where $r_{i j}$ are defined by

$$
\begin{aligned}
& r_{12}=\sum r_{1} \otimes r_{2} \otimes 1=r \otimes 1, \\
& r_{13}=\sum r_{1} \otimes 1 \otimes r_{2}=(1 \otimes \tau)(r \otimes 1)=(\tau \otimes 1)(1 \otimes r), \\
& r_{23}=\sum 1 \otimes r_{1} \otimes r_{2}=1 \otimes r
\end{aligned}
$$

and considered as elements in $U(\mathfrak{L})$, the universal enveloping algebra of a Lie superalgebra $\mathfrak{L}$. Elements (3.3) belongs to $\mathfrak{L} \otimes \mathfrak{L} \otimes \mathfrak{L}$.

Definition 3.4. The classical Hom-Yang-Baxter equation $(\mathrm{CHYBE})$ in a Hom-Lie superalgebra $(\mathfrak{L},[\cdot, \cdot], \alpha)$ is

$$
[[r, r]]^{\alpha}=\left[r_{12}, r_{13}\right]+\left[r_{12}, r_{23}\right]+\left[r_{13}, r_{23}\right]=0
$$


for $r \in \mathfrak{L} \otimes \mathfrak{L}$. The three brackets in (3.4) are defined as

$$
\begin{aligned}
& {\left[r_{12}, r_{13}^{\prime}\right]=\sum(-1)^{\left|r_{1}^{\prime}\right|\left|r_{2}\right|}\left[r_{1}, r_{1}^{\prime}\right] \otimes \alpha\left(r_{2}\right) \otimes \alpha\left(r_{2}^{\prime}\right),} \\
& {\left[r_{12}, r_{23}^{\prime}\right]=\sum \alpha\left(r_{1}\right) \otimes\left[r_{2}, r_{1}^{\prime}\right] \otimes \alpha\left(r_{2}^{\prime}\right),} \\
& {\left[r_{13}, r_{23}^{\prime}\right]=\sum(-1)^{\left|r_{1}^{\prime}\right|\left|r_{2}\right|} \alpha\left(r_{1}\right) \otimes \alpha\left(r_{1}^{\prime}\right) \otimes\left[r_{2}, r_{2}^{\prime}\right]}
\end{aligned}
$$

where $r=\sum r_{1} \otimes r_{2}$ and $r^{\prime}=\sum r_{1}^{\prime} \otimes r_{2}^{\prime} \in \mathfrak{L} \otimes \mathfrak{L}$. If $\alpha=I d$, then the (CHYBE) reduces to the (CYBE).

Definition 3.5. A (multiplicative) quasi-triangular Hom-Lie superbialgebra is a (multiplicative) coboundary Hom-Lie superbialgebra in which $r$ is a solution of the CHYBE (3.4). In these cases, we also write $\Delta$ as $a d(r)$.

Remark 3.6. Note that we do not require $r$ to be skew-supersymmetric in a coboundary Hom-Lie superbialgebra, whereas in [8] $r$ is assumed to be skew-supersymmetric in a coboundary Lie bialgebra. We follow the convention in [16] and 27].

Remark 3.7. Condition (3.1) is a natural because from Remark 1.8 the compatibility condition (1.8) in HomLie superbialgebra $\mathfrak{L}$ says that the cobracket $\Delta$ is a 1 -cocycle in $C^{1}\left(\mathfrak{L}, \mathfrak{L}^{\otimes 2}\right)$, where $\mathfrak{L}$ acts on $\mathfrak{L}^{\otimes 2}$ via the $\alpha$-twisted adjoint action (1.9). The simplest 1-cocycles are the 1-coboundaries, i.e, images of $\delta_{H L}^{0}$. We can define the Hom-Lie 0 -cochains and 0 th differential as follows, extending the definition in $\left[20\right.$. Set $C^{0}\left(\mathfrak{L}, \mathfrak{L}^{\otimes 2}\right)$ as the subspace of $\mathfrak{L}^{\otimes 2}$ consisting of elements that are fixed by $\alpha^{\otimes 2}$. Then we define the differential

$$
\delta_{H L}^{0}: C^{0}\left(\mathfrak{L}, \mathfrak{L}^{\otimes 2}\right) \rightarrow C^{1}\left(\mathfrak{L}, \mathfrak{L}^{\otimes 2}\right)
$$

by setting $\delta_{H L}^{0}(r)=a d(r)$, as in (1.6). It is not hard to check that, for $r \in C^{0}\left(\mathfrak{L}, \mathfrak{L}^{\otimes 2}\right)$, we have $\delta_{H L}^{1}\left(\delta_{H L}^{0}(r)\right)=0$, where $\delta_{H L}^{1}$ is defined in (1.10). In fact, what this condition says is that

$$
\begin{aligned}
0 & =\delta_{H L}^{1}\left(\delta_{H L}^{0}(r)\right)(x, y) \\
& =\delta_{H L}^{1}(a d(r))(x, y)=a d_{[x, y]}(r)-a d_{\alpha(x)}\left(a d_{y}(r)\right)+(-1)^{|x||y|} a d_{\alpha(y)}\left(a d_{x}(r)\right)
\end{aligned}
$$

for all $x, y \in \mathfrak{L}$. We will prove (3.6) in Lemma 3.11 below. Thus, such an element $\delta_{H L}^{0}(r)=a d(r)$ is a 1-coboundary, and hence a 1-cocycle. This fact makes $a d(r)$ (with $\alpha^{\otimes 2}(r)=r$ ) a natural candidate for the cobracket in a Hom-Lie superbialgebra and also justifies the name coboundary Hom-Lie superbialgebra.

The following result is the analogue of Theorem 1.11 for coboundary or quasi-triangular Hom-Lie superbialgebras. It says that coboundary or quasi-triangular Hom-Lie superbialgebras deform into other coboundary or quasi-triangular Hom-Lie superbialgebras via suitable endomorphisms.

Theorem 3.8. Let $(\mathfrak{L},[\cdot, \cdot], \Delta=a d(r), \alpha, r)$ be a coboundary Hom-Lie superbialgebra and an even map $\beta: \mathfrak{L} \rightarrow \mathfrak{L}$ be a morphism such that $\beta^{\otimes 2}(r)=r$. Then $\mathfrak{L}_{\beta}=\left(\mathfrak{L},[\cdot, \cdot]_{\beta}=\beta \circ[\cdot, \cdot], \Delta_{\beta}=\Delta \circ \beta, \beta \alpha, r\right)$ is also a coboundary Hom-Lie superbialgebra, which is multiplicative if $\mathfrak{L}$ is. Moreover, if $\mathfrak{L}$ is quasi-triangular, then so is $\mathfrak{L}_{\beta}$.

Proof. By Theorem 1.11 we know that $\mathfrak{L}_{\beta}$ is a Hom-Lie superbialgebra, multiplicative if $\mathfrak{L}$ is. To check that $\mathfrak{L}_{\beta}$ is coboundary, first note that $(\beta \alpha)^{\otimes 2}(r)=\beta^{\otimes 2} \alpha^{\otimes 2}(r)=r$.

To check the condition (3.1) in $\mathfrak{L}_{\beta}$, we compute as follows:

$$
\begin{aligned}
\Delta_{\beta}(x) & =\beta^{\otimes 2}(\Delta(x))=\beta^{\otimes 2}\left(\left[x, r_{1}\right] \otimes \alpha\left(r_{2}\right)\right)+\beta^{\otimes 2}\left((-1)^{|x|\left|r_{1}\right|} \alpha\left(r_{1}\right) \otimes\left[x, r_{2}\right]\right) \\
& =\left[x, r_{1}\right]_{\beta} \otimes \beta \alpha\left(r_{2}\right)+(-1)^{|x|\left|r_{1}\right|} \beta \alpha\left(r_{1}\right) \otimes\left[x, r_{2}\right]_{\beta} .
\end{aligned}
$$

The last expression above is $a d_{x}(r)$ in $\mathfrak{L}_{\beta}$, which shows that $\mathfrak{L}_{\beta}$ is coboundary.

Finally, suppose in addition that $\mathfrak{L}$ is quasi-triangular, i.e., $r$ is a solution of the CHYBE in $\mathfrak{L}$. Using the notation in (3.4) we have:

$$
0=\beta^{\otimes 3}\left(\left[r_{12}, r_{13}\right]+\left[r_{12}, r_{23}\right]+\left[r_{13}, r_{23}\right]\right)=\left[r_{12}, r_{13}\right]_{\beta}+\left[r_{12}, r_{23}\right]_{\beta}+\left[r_{13}, r_{23}\right]_{\beta},
$$

where the last expression is defined in $\mathfrak{L}_{\beta}$. This shows that $r$ is a solution of the CHYBE in $\mathfrak{L}_{\beta}$, so $\mathfrak{L}_{\beta}$ is quasi-triangular.

The following result is the analogue of Corollary 1.12 for coboundary or quasi-triangular Hom-Lie superbialgebras. It says that these objects can be obtained by twisting coboundary or quasi-triangular Lie superbialgebras via suitable endomorphisms. 
Corollary 3.9. Let $(\mathfrak{L},[\cdot, \cdot], \Delta, r)$ be a coboundary Lie superbialgebra and an even map $\beta: \mathfrak{L} \rightarrow \mathfrak{L}$ be a Lie superalgebra morphism such that $\beta^{\otimes 2}(r)=r$. Then $\mathfrak{L}_{\beta}=\left(\mathfrak{L},[\cdot, \cdot]_{\beta}=\beta \circ[\cdot, \cdot], \Delta_{\beta}=\Delta \circ \beta, \beta, r\right)$ is a multiplicative coboundary Hom-Lie superbialgebra. If, in addition, $\mathfrak{L}$ is a quasi-triangular Lie superbialgebra, then $\mathfrak{L}_{\beta}$ is a multiplicative quasi-triangular Hom-Lie superbialgebra.

Proof. This is the $\alpha=I d$ special case of Theorem 3.8. provided that we can show that $\Delta \circ \beta=\beta^{\otimes 2} \circ \Delta$. We compute as follows:

$$
\begin{aligned}
\beta^{\otimes 2}(\Delta(x)) & =\beta^{\otimes 2}\left(a d_{x}(r)\right)=\beta^{\otimes 2}\left(\left[x, r_{1}\right] \otimes r_{2}\right)+\beta^{\otimes 2}\left((-1)^{|x|\left|r_{1}\right|} r_{1} \otimes\left[x, r_{2}\right]\right) \\
& =\left[\beta(x), \beta\left(r_{1}\right)\right] \otimes \beta\left(r_{2}\right)+(-1)^{|x|\left|r_{1}\right|} \beta\left(r_{1}\right) \otimes\left[\beta(x), \beta\left(r_{2}\right)\right] \\
& =\left[\beta(x), r_{1}\right] \otimes r_{2}+(-1)^{|x|\left|r_{1}\right|} r_{1} \otimes\left[\beta(x), r_{2}\right]=a d_{\beta(x)}(r)=\Delta(\beta(x)) .
\end{aligned}
$$

The next result says that every multiplicative coboundary or quasi-triangular Hom-Lie superbialgebra gives rise to an infinite sequence of multiplicative coboundary or quasi-triangular Hom-Lie superbialgebra. It is similar to Corollary 1.13 .

Corollary 3.10. Let $(\mathfrak{L},[\cdot, \cdot], \Delta, \alpha, r)$ be a multiplicative coboundary (resp. quasi-triangular) Hom-Lie superbialgebra. Then $\mathfrak{L}_{\alpha^{n}}=\left(\mathfrak{L},[\cdot, \cdot]_{\alpha^{n}}=\alpha^{n} \circ[\cdot, \cdot], \Delta_{\alpha^{n}}=\Delta \circ \alpha^{n}, \alpha^{n+1}, r\right)$ is also a multiplicative coboundary (resp. quasi-triangular) Hom-Lie superbialgebra for each integer $n \geq 0$.

Proof. This is the $\beta=\alpha^{n}$ special case of Theorem 3.8 .

In the following result, we describe some sufficient condition under which a Hom-Lie superalgebra becomes a coboundary Hom-Lie superbialgebra.

In what follows, for an element $r=\sum r_{1} \otimes r_{2}$, we write $r_{21}$ for $\tau(r)=\sum(-1)^{\left|r_{1}\right|\left|r_{2}\right|} r_{2} \otimes r_{1}$.

Lemma 3.11. Let $(\mathfrak{L},[\cdot, \cdot], \alpha)$ be a multiplicative Hom-Lie superalgebra and $r \in \mathfrak{L}^{\otimes 2}$ be an element such that $\alpha^{\otimes 2}(r)=r$, (1.3) and (3.2), (3.2) and (1.3), i.e., $\left.|r|=\overline{0}\right)$. Then $\Delta=\operatorname{ad}(r): \mathfrak{L} \rightarrow \mathfrak{L} \otimes 2$ satisfies (1.8), i.e., $a d_{[x, y]}(r)=a d_{\alpha(x)}\left(a d_{y}(r)\right)-(-1)^{|x||y|} a d_{\alpha(y)}\left(a d_{x}(r)\right)$ for $x, y \in \mathfrak{L}$.

Proof. We will use $\alpha^{\otimes 2}(r)=r$, the skew-supersymmetry (1.1) and the Hom-Jacobi identity of (1.2) and $\alpha([x, y])=[\alpha(x), \alpha(y)]$, (multiplicative) in the computation below. For $x, y \in \mathfrak{L}$, we have:

$$
\begin{aligned}
a d_{[x, y]}(r) & =\left[[x, y], r_{1}\right] \otimes \alpha\left(r_{2}\right)+(-1)^{|[x, y]|\left|r_{1}\right|} \alpha\left(r_{1}\right) \otimes\left[[x, y], r_{2}\right] \\
& =\left[[x, y], \alpha\left(r_{1}\right)\right] \otimes \alpha^{2}\left(r_{2}\right)+(-1)^{|x|\left|r_{1}\right|}(-1)^{|y|\left|r_{1}\right|} \alpha^{2}\left(r_{1}\right) \otimes\left[[x, y], \alpha\left(r_{2}\right)\right] \\
& =\left(\left[\alpha(x),\left[y, r_{1}\right]\right]+(-1)^{|x||y|}(-1)^{|x|\left|r_{1}\right|}\left[\alpha(y),\left[r_{1}, x\right]\right]\right) \otimes \alpha^{2}\left(r_{2}\right) \\
& +(-1)^{|x|\left|r_{1}\right|}(-1)^{|y|\left|r_{1}\right|} \alpha^{2}\left(r_{1}\right) \otimes\left(\left[\alpha(x),\left[y, r_{2}\right]\right]+(-1)^{|x||y|}(-1)^{|x|\left|r_{2}\right|}\left[\alpha(y),\left[r_{2}, x\right]\right]\right) \\
& =\left[\alpha(x),\left[y, r_{1}\right]\right] \otimes \alpha^{2}\left(r_{2}\right)+(-1)^{|x||y|}(-1)^{|x|\left|r_{1}\right|}\left[\alpha(y),\left[r_{1}, x\right]\right] \otimes \alpha^{2}\left(r_{2}\right) \\
& +(-1)^{|x|\left|r_{1}\right|}(-1)^{|y|\left|r_{1}\right|} \alpha^{2}\left(r_{1}\right) \otimes\left[\alpha(x),\left[y, r_{2}\right]\right]+(-1)^{|x||y|}(-1)^{|y|\left|r_{1}\right|} \alpha^{2}\left(r_{1}\right) \otimes\left[\alpha(y),\left[r_{2}, x\right]\right] \\
& =\left[\alpha(x),\left[y, r_{1}\right]\right] \otimes \alpha^{2}\left(r_{2}\right)+(-1)^{|x||y|}(-1)^{|x|\left|r_{1}\right|} \alpha\left(\left[y, r_{1}\right]\right) \otimes\left[\alpha(x), \alpha\left(r_{2}\right)\right] \\
& +(-1)^{|y|\left|r_{1}\right|}\left[\alpha(x), \alpha\left(r_{1}\right)\right] \otimes \alpha\left(\left[y, r_{2}\right]\right)+(-1)^{|x|\left|r_{1}\right|}(-1)^{|y|\left|r_{1}\right|} \alpha^{2}\left(r_{1}\right) \otimes\left[\alpha(x),\left[y, r_{2}\right]\right] \\
& -(-1)^{|x||y|}\left[\alpha(y),\left[x, r_{1}\right]\right] \otimes \alpha^{2}\left(r_{2}\right)-(-1)^{|y|\left|r_{1}\right|} \alpha\left(\left[x, r_{1}\right]\right) \otimes\left[\alpha(y), \alpha\left(r_{2}\right)\right] \\
& -(-1)^{|x||y|}(-1)^{|x|\left|r_{1}\right|}\left[\alpha(y), \alpha\left(r_{1}\right)\right] \otimes \alpha\left(\left[x, r_{2}\right]\right)-(-1)^{|x||y|}(-1)^{|y|\left|r_{1}\right|+|x|\left|r_{1}\right|} \alpha^{2}\left(r_{1}\right) \otimes\left[\alpha(y),\left[x, r_{2}\right]\right] \\
& =a d_{\alpha(x)}\left(\left[y, r_{1}\right] \otimes \alpha\left(r_{2}\right)+(-1)^{|y|\left|r_{1}\right|} \alpha\left(r_{1}\right) \otimes\left[y, r_{2}\right]\right) \\
& -(-1)^{|x||y|} a d_{\alpha(y)}\left(\left[x, r_{1}\right] \otimes \alpha\left(r_{2}\right)+(-1)^{|x|\left|r_{1}\right|} \alpha\left(r_{1}\right) \otimes\left[x, r_{2}\right]\right) \\
& =a d_{\alpha(x)}\left(a d_{y}(r)\right)-(-1)^{|x||y|} a d_{\alpha(y)}\left(a d_{x}(r)\right) .
\end{aligned}
$$

Theorem 3.12. Let $(\mathfrak{L},[\cdot, \cdot], \alpha)$ be a multiplicative Hom-Lie superalgebra and $r \in \mathfrak{L} \otimes 2$ be an element such that $\alpha^{\otimes 2}(r)=r, \quad r_{21}=-r,(1.3)$ and (3.2), (3.2) and (1.3), i.e., $\left.|r|=\overline{0}\right)$, and

$$
\alpha^{\otimes 3}\left(a d_{x}\left([[r, r]]^{\alpha}\right)\right)=0
$$


for all $x \in \mathfrak{L}$, where $[[r, r]]^{\alpha}$ is defined in (3.4). Define $\Delta: \mathfrak{L} \rightarrow \mathfrak{L}^{\otimes 2}$ as $\Delta(x)=a d_{x}(r)$ as in (3.1).

Then $(\mathfrak{L},[\cdot, \cdot], \Delta, \alpha, r)$ is a multiplicative coboundary Hom-Lie superbialgebra.

Proof. We will show the following statements:

1) $\Delta=\operatorname{ad}(r)$ commutes with $\alpha$.

2) $\Delta$ is skew-supersymmetric.

3) The compatibility condition (1.8) holds.

4) The condition (3.7) is equivalent Hom-super-coJacobi identity of $\Delta$ (1.5).

Write $r=\sum r_{1} \otimes r_{2}, r^{\prime}=\sum r_{1}^{\prime} \otimes r_{2}^{\prime}$ and $\tau(r)=\sum(-1)^{\left|r_{1}\right|\left|r_{2}\right|} r_{2} \otimes r_{1}, \tau\left(r^{\prime}\right)=\sum(-1)^{\left|r_{1}^{\prime}\right|\left|r_{2}^{\prime}\right|} r_{2}^{\prime} \otimes r_{1}^{\prime}$. To show that $\Delta=a d(r)$ commutes with $\alpha$, pick an element $x \in \mathfrak{L}$, the summation sign will often be omitted in computation to simplify the typography. Using the definition $\Delta=\operatorname{ad}(r), \alpha\left(\left[x, r_{1}\right]\right)=\left[\alpha(x), \alpha\left(r_{1}\right)\right]$, $\alpha\left(\left[x, r_{2}\right]\right)=\left[\alpha(x), \alpha\left(r_{2}\right)\right]$ and the assumption $\alpha^{\otimes 2}(r)=r$ we have

$$
\begin{aligned}
\Delta(\alpha(x)) & =\left[\alpha(x), r_{1}\right] \otimes \alpha\left(r_{2}\right)+(-1)^{|x|\left|r_{1}\right|} \alpha\left(r_{1}\right) \otimes\left[\alpha(x), r_{2}\right] \\
& =\alpha\left(\left[x, r_{1}\right]\right) \otimes \alpha^{2}\left(r_{2}\right)+(-1)^{|x|\left|r_{1}\right|} \alpha^{2}\left(r_{1}\right) \otimes \alpha\left(\left[x, r_{2}\right]\right)=\alpha^{\otimes 2}(\Delta(x)) .
\end{aligned}
$$

This shows that $\Delta$ commutes with $\alpha$.

Now we show that $\Delta=a d(r)$ is skew-supersymmetric. We have

$$
\begin{aligned}
\Delta(x) & =\left[x, r_{1}\right] \otimes \alpha\left(r_{2}\right)+(-1)^{|x|\left|r_{1}\right|} \alpha\left(r_{1}\right) \otimes\left[x, r_{2}\right] . \\
\tau(\Delta(x)) & =(-1)^{\left|r_{1}\right|\left|r_{2}\right|}\left(\left[x, r_{2}\right] \otimes \alpha\left(r_{1}\right)+(-1)^{|x|\left|r_{2}\right|} \alpha\left(r_{2}\right) \otimes\left[x, r_{1}\right]\right) .
\end{aligned}
$$

Then $\Delta(x)+\tau(\Delta(x))=a d_{x}\left(r+r_{21}\right)=a d_{x}(0)=0$, since

$$
r+r_{21}=\sum\left(r_{1} \otimes r_{2}+(-1)^{\left|r_{1}\right|\left|r_{2}\right|} r_{2} \otimes r_{1}\right) .
$$

We will prove that the compatibility condition (1.8) holds in Lemma 3.11

Finally, we show that the Hom-super-coJacobi identity (1.5) of $\Delta=\operatorname{ad}(r)$ is equivalent to (3.7). Let us unwrap the Hom-super-coJacobi identity. Fix an element $x \in \mathfrak{L}$, and let $r^{\prime}=\sum r_{1}^{\prime} \otimes r_{2}^{\prime}$ be another copy of $r$. Then we write

$$
\begin{aligned}
\omega & =(\alpha \otimes \Delta)(\Delta(x)) \\
& =(\alpha \otimes \Delta)\left(\left[x, r_{1}\right] \otimes \alpha\left(r_{2}\right)+(-1)^{|x|\left|r_{1}\right|} \alpha\left(r_{1}\right) \otimes\left[x, r_{2}\right]\right) \\
& =\alpha\left(\left[x, r_{1}\right]\right) \otimes\left[\alpha\left(r_{2}\right), r_{1}^{\prime}\right] \otimes \alpha\left(r_{2}^{\prime}\right)+(-1)^{\left|r_{2}\right|\left|r_{1}^{\prime}\right|} \alpha\left(\left[x, r_{1}\right]\right) \otimes \alpha\left(r_{1}^{\prime}\right) \otimes\left[\alpha\left(r_{2}\right), r_{2}^{\prime}\right] \\
& +(-1)^{|x|\left|r_{1}\right|} \alpha^{2}\left(r_{1}\right) \otimes\left[\left[x, r_{2}\right], r_{1}^{\prime}\right] \otimes \alpha\left(r_{2}^{\prime}\right)+(-1)^{|x|\left|r_{1}\right|}(-1)^{|x|\left|r_{1}^{\prime}\right|}(-1)^{\left|r_{2}\right|\left|r_{1}^{\prime}\right|} \alpha^{2}\left(r_{1}\right) \otimes \alpha\left(r_{1}^{\prime}\right) \otimes\left[\left[x, r_{2}\right], r_{2}^{\prime}\right] .
\end{aligned}
$$

Note

$$
\begin{aligned}
& A_{1}=\alpha\left(\left[x, r_{1}\right]\right) \otimes\left[\alpha\left(r_{2}\right), r_{1}^{\prime}\right] \otimes \alpha\left(r_{2}^{\prime}\right), \\
& B_{1}=(-1)^{\left|r_{2}\right|\left|r_{1}^{\prime}\right|} \alpha\left(\left[x, r_{1}\right]\right) \otimes \alpha\left(r_{1}^{\prime}\right) \otimes\left[\alpha\left(r_{2}\right), r_{2}^{\prime}\right], \\
& C_{1}=(-1)^{|x|\left|r_{1}\right|} \alpha^{2}\left(r_{1}\right) \otimes\left[\left[x, r_{2}\right], r_{1}^{\prime}\right] \otimes \alpha\left(r_{2}^{\prime}\right), \\
& D_{1}=(-1)^{|x|\left|r_{1}\right|}(-1)^{|x|\left|r_{1}^{\prime}\right|}(-1)^{\left|r_{2}\right|\left|r_{1}^{\prime}\right|} \alpha^{2}\left(r_{1}\right) \otimes \alpha\left(r_{1}^{\prime}\right) \otimes\left[\left[x, r_{2}\right], r_{2}^{\prime}\right] .
\end{aligned}
$$

we get $\omega=A_{1}+B_{1}+C_{1}+D_{1}$.

With these notations, the Hom-super-coJacobi identity of $\Delta=a d(r)$ (applied to $x$ ) becomes

$$
\left(1 \otimes 1 \otimes 1+\xi+\xi^{2}\right) \circ(\alpha \otimes \Delta) \circ \Delta(x)=\sum_{i=1}^{3}\left(A_{i}+B_{i}+C_{i}+D_{i}\right)=0 .
$$

Therefore, to prove the equivalence between the Hom-super-coJacobi identity of $\Delta$ and (3.7), it suffices to show

$$
\alpha^{\otimes 3}\left(a d_{x}\left([[r, r]]^{\alpha}\right)\right)=\sum_{i=1}^{3}\left(A_{i}+B_{i}+C_{i}+D_{i}\right),
$$

which we will prove in Lemma 3.13 below.

The proof of Theorem 3.12 will be completed once we prove the Lemma below. 
Lemma 3.13. The condition (3.9) holds.

Proof. It suffices to show the following three equalities:

$$
\begin{gathered}
\alpha^{\otimes 3}\left(a d_{x}\left(\left[r_{12}, r_{13}\right]\right)\right)=A_{3}+B_{2}+C_{3}+D_{2}, \\
\alpha^{\otimes 3}\left(a d_{x}\left(\left[r_{12}, r_{23}\right]\right)\right)=A_{1}+B_{3}+C_{1}+D_{3}, \\
\alpha^{\otimes 3}\left(a d_{x}\left(\left[r_{13}, r_{23}\right]\right)\right)=A_{2}+B_{1}+C_{2}+D_{1},
\end{gathered}
$$

where the three bracket, which add up to $[[r, r]]^{\alpha}$, are defined in (3.5). The proofs for the three equalities are very similar, so we will only give the proof of (3.10).

Since $r=r^{\prime}$ and $r_{12}=-r$, we have

$$
\begin{aligned}
A_{3} & =(-1)^{|x|\left|r_{2}\right|}(-1)^{\left|r_{1}^{\prime}\right|\left|r_{1}\right|}\left[\alpha\left(r_{2}\right), r_{1}^{\prime}\right] \otimes \alpha\left(r_{2}^{\prime}\right) \otimes \alpha\left(\left[x, r_{1}\right]\right) \\
& =(-1)^{|x|\left|r_{2}\right|}(-1)^{\left|r_{1}\right|\left|r_{1}^{\prime}\right|}\left[\alpha\left(r_{2}^{\prime}\right), r_{1}\right] \otimes \alpha\left(r_{2}\right) \otimes \alpha\left(\left[x, r_{1}^{\prime}\right]\right) \\
& =-(-1)^{|x|\left|r_{2}\right|}\left[\alpha\left(r_{1}^{\prime}\right), r_{1}\right] \otimes \alpha\left(r_{2}\right) \otimes \alpha\left(\left[x, r_{2}^{\prime}\right]\right) \\
& =-(-1)^{|x|\left|r_{2}\right|}\left[\alpha^{2}\left(r_{1}^{\prime}\right), \alpha^{2}\left(r_{1}\right)\right] \otimes \alpha^{3}\left(r_{2}\right) \otimes \alpha\left(\left[x, \alpha\left(r_{2}^{\prime}\right)\right]\right) \\
& =\alpha^{\otimes 3}\left((-1)^{|x|\left|r_{2}\right|}(-1)^{\left|r_{1}^{\prime}\right|\left|r_{2}\right|} \alpha\left(\left[r_{1}, r_{1}^{\prime}\right]\right) \otimes \alpha^{2}\left(r_{2}\right) \otimes\left[x, \alpha\left(r_{2}^{\prime}\right)\right]\right) .
\end{aligned}
$$

In the fourth equality we used $\alpha^{\otimes 2}(r)=r, \alpha^{\otimes 2}\left(r^{\prime}\right)=r^{\prime}$. In the equality we used the skew-supersymmetry of $[-,-]$ and $\alpha\left(\left[r_{1}, r_{1}^{\prime}\right]\right)=\left[\alpha\left(r_{1}\right), \alpha\left(r_{1}^{\prime}\right)\right]$, we know $(-1)^{|x|\left|r_{1}\right|}(-1)^{|x|\left|r_{1}^{\prime}\right|}=1$, just to check the calculations. Similar computation give

$$
\begin{gathered}
B_{2}=(-1)^{\left|r_{1}^{\prime}\right|\left|r_{1}\right|}\left[\alpha\left(r_{2}\right), r_{2}^{\prime}\right] \otimes \alpha\left(\left[x, r_{1}\right]\right) \otimes \alpha\left(r_{1}^{\prime}\right) \\
=\alpha^{\otimes 3}\left((-1)^{|x|\left|r_{1}\right|}(-1)^{|x|\left|r_{1}^{\prime}\right|}(-1)^{\left|r_{1}^{\prime}\right|\left|r_{2}\right|} \alpha\left(\left[r_{1}, r_{1}^{\prime}\right]\right) \otimes\left[x, \alpha\left(r_{2}\right)\right] \otimes \alpha^{2}\left(r_{2}^{\prime}\right)\right), \\
C_{3}=(-1)^{\left|r_{1}^{\prime}\right|\left|r_{2}\right|}\left[\left[x, r_{2}\right], r_{1}^{\prime}\right] \otimes \alpha\left(r_{2}^{\prime}\right) \otimes \alpha^{2}\left(r_{1}\right)=(-1)^{|x|\left|r_{2}^{\prime}\right|}\left[\left[r_{2}^{\prime}, x\right], \alpha\left(r_{2}\right)\right] \otimes \alpha^{2}\left(r_{1}\right) \otimes \alpha^{2}\left(r_{1}^{\prime}\right), \\
D_{2}=(-1)^{\left|r_{1}^{\prime}\right|\left|r_{2}\right|}\left[\left[x, r_{2}\right], r_{2}^{\prime}\right] \otimes \alpha^{2}\left(r_{2}^{\prime}\right) \otimes \alpha\left(r_{1}^{\prime}\right)=(-1)^{\left|r_{1}^{\prime}\right|\left|r_{2}\right|}\left[\left[x, r_{2}\right], \alpha\left(r_{2}^{\prime}\right)\right] \otimes \alpha^{2}\left(r_{1}\right) \otimes \alpha^{2}\left(r_{1}^{\prime}\right) .
\end{gathered}
$$

Using, in addition, the skew-supersymmetry (1.1) and the Hom-Jacobi identity (1.2) of [-, - ], we add $C_{3}$ and $D_{2}$ :

$$
\begin{aligned}
C_{3}+D_{2} & =(-1)^{\left|r_{1}^{\prime}\right|\left|r_{2}\right|}\left((-1)^{|x|\left|r_{2}^{\prime}\right|}(-1)^{\left|r_{2}\right|\left|r_{2}^{\prime}\right|}\left[\left[r_{2}^{\prime}, x\right], \alpha\left(r_{2}\right)\right]+\left[\left[x, r_{2}\right], \alpha\left(r_{2}^{\prime}\right)\right]\right) \otimes \alpha^{2}\left(r_{1}\right) \otimes \alpha^{2}\left(r_{1}^{\prime}\right) \\
& =(-1)^{\left|r_{1}^{\prime}\right|\left|r_{2}\right|}\left[\alpha(x),\left[r_{2}, r_{2}^{\prime}\right]\right] \otimes \alpha^{2}\left(r_{1}\right) \otimes \alpha^{2}\left(r_{1}^{\prime}\right) \\
& =(-1)^{\left|r_{1}^{\prime}\right|\left|r_{2}\right|}\left[\alpha(x),\left[r_{1}, r_{1}^{\prime}\right]\right] \otimes \alpha^{2}\left(r_{2}\right) \otimes \alpha^{2}\left(r_{2}^{\prime}\right) \\
& =(-1)^{\left|r_{1}^{\prime}\right|\left|r_{2}\right|}\left[\alpha(x),\left[\alpha\left(r_{1}\right), \alpha\left(r_{1}^{\prime}\right)\right]\right] \otimes \alpha^{3}\left(r_{2}\right) \otimes \alpha^{3}\left(r_{2}^{\prime}\right) \\
& =\alpha^{\otimes 3}\left((-1)^{\left|r_{1}^{\prime}\right|\left|r_{2}\right|}\left[x,\left[r_{1}, r_{1}^{\prime}\right]\right] \otimes \alpha^{2}\left(r_{2}\right) \otimes \alpha^{2}\left(r_{2}^{\prime}\right)\right) .
\end{aligned}
$$

Using the definition (1.6) of $a d_{x}$, we now conclude that:

$$
\begin{aligned}
A_{3}+B_{2}+C_{3}+D_{2} & =\alpha^{\otimes 3}\left((-1)^{|x|\left|r_{2}\right|}(-1)^{\left|r_{1}^{\prime}\right|\left|r_{2}\right|} \alpha\left(\left[r_{1}, r_{1}^{\prime}\right]\right) \otimes \alpha^{2}\left(r_{2}\right) \otimes\left[x, \alpha\left(r_{2}^{\prime}\right)\right]\right) \\
& +\alpha^{\otimes 3}\left((-1)^{|x|\left|r_{1}\right|}(-1)^{|x|\left|r_{1}^{\prime}\right|}(-1)^{\left|r_{1}^{\prime}\right|\left|r_{2}\right|} \alpha\left(\left[r_{1}, r_{1}^{\prime}\right]\right) \otimes\left[x, \alpha\left(r_{2}\right)\right] \otimes \alpha^{2}\left(r_{2}^{\prime}\right)\right) \\
& +\alpha^{\otimes 3}\left((-1)^{\left|r_{1}^{\prime}\right|\left|r_{2}\right|}\left[x,\left[r_{1}, r_{1}^{\prime}\right]\right] \otimes \alpha^{2}\left(r_{2}\right) \otimes \alpha^{2}\left(r_{2}^{\prime}\right)\right) \\
& =\alpha^{\otimes 3}\left((-1)^{\left|r_{1}^{\prime}\right|\left|r_{2}\right|} a d_{x}\left(\left[r_{1}, r_{1}^{\prime}\right] \otimes \alpha\left(r_{2}\right) \otimes \alpha\left(r_{2}^{\prime}\right)\right)\right) \\
& =\alpha^{\otimes 3}\left(a d_{x}\left((-1)^{\left|r_{1}^{\prime}\right|\left|r_{2}\right|}\left[r_{1}, r_{1}^{\prime}\right] \otimes \alpha\left(r_{2}\right) \otimes \alpha\left(r_{2}^{\prime}\right)\right)\right) \\
& =\alpha^{\otimes 3}\left(a d_{x}\left(\left[r_{12}, r_{13}\right]\right)\right) .
\end{aligned}
$$

This proves (3.10).

The equalities (3.11) and (3.12) are proved by very similar computations.

Therefore, the equality (3.9) holds. Together with (3.8) we have shown that the the Hom-super-coJacobi identity of $\Delta=a d(r)$ is equivalent to $\alpha^{\otimes 3}\left(a d_{x}\left([[r, r]]^{\alpha}\right)\right)=0$ 
The following result is an immediate consequence of Theorem (3.12). It gives sufficient conditions under which a Hom-Lie superalgebra becomes a quasi-triangular Hom-Lie superbialgebra.

Corollary 3.14. Let $(\mathfrak{L},[\cdot, \cdot], \alpha)$ be a multiplicative Hom-Lie superalgebra and $r \in \mathfrak{L} \otimes 2$ be an element such that $\alpha^{\otimes 2}(r)=r, \quad r_{21}=-r$, (1.3) and (3.2), (3.2) and (1.3), i.e., $|r|=\overline{0}$ ), and $[[r, r]]^{\alpha}=0$. Then $(\mathfrak{L},[\cdot, \cdot]$, ad $(r), \alpha, r)$ is a multiplicative quasi-triangular Hom-Lie superbialgebra.

Theorem 3.15. Let $(\mathfrak{L},[\cdot, \cdot], \Delta, \alpha, r)$ be a coboundary Hom-Lie superbialgebra. Then the following statements are equivalent,

(1) $\mathfrak{L}$ is a quasi-triangular Hom-Lie superbialgebra, i.e., $[[r, r]]^{\alpha}=0$ [3.4).

(2) The equality $(\alpha \otimes \Delta)(r)=-\left[r_{12}, r_{13}\right]$ holds, where the bracket is defined in (3.5).

(3) The equality $(\Delta \otimes \alpha)(r)=\left[r_{13}, r_{23}\right]$ holds, where the bracket is defined in (3.5).

Proof. The equivalence between three statements clearly follows from the equalities. Let $r^{\prime}=\sum r_{1}^{\prime} \otimes r_{2}^{\prime}$ be another copy of $r$. Since $\Delta=a d(r)$ (3.1),$r=r^{\prime}$ and $r_{1} \otimes r_{2}=-(-1)^{\left|r_{1}\right|\left|r_{2}\right|} r_{2} \otimes r_{1}$. By calculation we will find results

$$
\begin{aligned}
(\alpha \otimes \Delta)\left(r_{1} \otimes r_{2}\right) & =\alpha\left(r_{1}\right) \otimes \Delta\left(r_{2}\right)=\alpha\left(r_{1}\right) \otimes\left[r_{2}, r_{1}^{\prime}\right] \otimes \alpha\left(r_{2}^{\prime}\right)+(-1)^{\left|r_{1}^{\prime}\right|\left|r_{2}\right|} \alpha\left(r_{1}\right) \otimes \alpha\left(r_{1}^{\prime}\right) \otimes\left[r_{2}, r_{2}^{\prime}\right] \\
& =\left[r_{12}, r_{23}\right]+\left[r_{13}, r_{23}\right] .
\end{aligned}
$$

This shows the equivalence between statements (1) and (2). Likewise, we have

$$
\begin{aligned}
(\Delta \otimes \alpha)\left(r_{1} \otimes r_{2}\right) & =\Delta\left(r_{1}\right) \otimes \alpha\left(r_{2}\right)=\left[r_{1}, r_{1}^{\prime}\right] \otimes \alpha\left(r_{2}^{\prime}\right) \otimes \alpha\left(r_{2}\right)+(-1)^{\left|r_{1}\right|\left|r_{1}^{\prime}\right|} \alpha\left(r_{1}^{\prime}\right) \otimes\left[r_{1}, r_{2}^{\prime}\right] \otimes \alpha\left(r_{2}\right) \\
& =-(-1)^{\left|r_{1}^{\prime}\right|\left|r_{2}\right|}\left[r_{1}, r_{1}^{\prime}\right] \otimes \alpha\left(r_{2}\right) \otimes \alpha\left(r_{2}^{\prime}\right)-\alpha\left(r_{1}\right) \otimes\left[r_{2}, r_{1}^{\prime}\right] \otimes \alpha\left(r_{2}^{\prime}\right) \\
& =-\left[r_{12}, r_{13}\right]-\left[r_{12}, r_{23}\right]
\end{aligned}
$$

This shows the equivalence between statements (1) and (3).

\section{Cobracket perturbation in Hom-Lie superbialgebras}

The purpose of this section is to study perturbation of cobrackets in Hom-Lie superbialgebras, following Drinfel'd's perturbation theory of quasi-Hopf algebras (6], 9], [10, [11]).

We address the following question : "If $(\mathfrak{L},[\cdot, \cdot], \Delta, \alpha)$ is a Hom-Lie superbialgebra (Definition 1.5) and $t \in \mathfrak{L}^{\otimes 2}$, under what conditions does the perturbed cobracket $\Delta_{t}=\Delta+a d(t)$ give another Hom-Lie superbialgebra $\left(\mathfrak{L},[\cdot, \cdot], \Delta_{t}, \alpha\right)$ ?"

Define the perturbed cobracket $\Delta_{t}=\Delta+a d(t)$. For $x \in \mathfrak{L}$ and $t=\sum t_{1} \otimes t_{2} \in \mathfrak{L}^{\otimes 2}$ and also recall the adjoint map $a d_{x}: \mathfrak{L}^{\otimes n} \rightarrow \mathfrak{L}^{\otimes n}(\underline{1.6}$ ) we have :

$$
\Delta_{t}(x)=\Delta(x)+a d_{x}(t)=\Delta(x)+a d_{x}\left(t_{1} \otimes t_{2}\right)=\Delta(x)+\left[x, t_{1}\right] \otimes \alpha\left(t_{2}\right)+(-1)^{|x|\left|t_{1}\right|} \alpha\left(t_{1}\right) \otimes\left[x, t_{2}\right] .
$$

This is a natural question because $\Delta$ is a 1-cocycle (Remark 1.8), $a d(t)$ (1.6) is a 1-coboundary when $\alpha^{\otimes 2}(t)=t$ (Remark 3.7), and perturbation of cocycles by coboundaries is a natural concept in homological algebra. Of course, we have more to worry about than just the cocycle condition (1.8) because $\left(\mathfrak{L}, \Delta_{t}, \alpha\right)$ must be a Hom-Lie supercoalgebra (Definition 1.2).

In the following result, we give some sufficient conditions under which the perturbed cobracket $\Delta_{t}$ gives another Hom-Lie superbialgebra. This is a generalization of [16], which deals with cobracket perturbation in Lie superbialgebras.

A result about cobracket perturbation in a quasi-triangular Hom-Lie superbialgebra, is given after the following result. We also briefly discuss triangular Hom-Lie superbialgebra, which is the Hom-Type version of Drinfel'd's triangular Lie bialgebra 8 .

Let us recall some notations first. For $t=\sum t_{1} \otimes t_{2} \in \mathfrak{L}^{\otimes 2}$, the symbol $t_{21}$ denotes $\tau(t)=\sum(-1)^{\left|t_{1}\right|\left|t_{2}\right|} t_{2} \otimes t_{1}$. If $\varphi(x, y)$ is an expression in the elements $x$ and $y$, we set

$$
|\varphi(x, y)|=\varphi(x, y)-(-1)^{|x||y|} \varphi(y, x)
$$


For example, the compatibility condition $\Delta([x, y])=a d_{\alpha(x)}(\Delta(y))-(-1)^{|x||y|} a d_{\alpha(y)}(\Delta(x))(1.8)$ is equivalent to

$$
\Delta([x, y])=\left|a d_{\alpha(x)}(\Delta(y))\right|
$$

Moreover $[[x, y], \alpha(z)]=|[\alpha(x),[y, z]]|$, where $|[\alpha(x),[y, z]]|=[\alpha(x),[y, z]]-(-1)^{|x||y|}[\alpha(y),[x, z]]$. By calculation the Hom-super-Jacobi identity (1.2) is equivalent to $[[x, y], \alpha(z)]=|[\alpha(x),[y, z]]|$. Note that we have

$$
|\varphi(x, y)+\psi(x, y)|=|\varphi(x, y)|+|\psi(x, y)|
$$

It is also noted to simplify writing $\left(1 \otimes 1 \otimes 1+\xi+\xi^{2}\right)=\circlearrowleft$.

Theorem 4.1. Let $(\mathfrak{L},[\cdot, \cdot], \Delta, \alpha)$ be a multiplicative Hom-Lie superbialgebra and $t \in \mathfrak{L}^{\otimes 2}$ be an element such that $\alpha^{\otimes 2}(t)=t, \quad t_{21}=-t$, (1.3) and (3.2), (3.2) and (1.3), i.e., $|t|=\overline{0}$ ) and

$$
\alpha^{\otimes 3}\left(a d_{x}\left([[t, t]]^{\alpha}+\circlearrowleft(\alpha \otimes \Delta)(t)\right)=0\right.
$$

for all $x \in \mathfrak{L}$. Then $\mathfrak{L}_{t}=\left(\mathfrak{L},[\cdot, \cdot], \Delta_{t}=\Delta+\operatorname{ad}(t), \alpha\right)$ is multiplicative Hom-Lie superbialgebra.

Proof. To show that $\mathfrak{L}_{t}$ is a multiplicative Hom-Lie superbialgebra, we need to prove the following conditions: It is clear that $(\mathfrak{L},[\cdot, \cdot], \alpha)$ is a multiplicative Hom-Lie superalgebra.

It remains to show that $\left(\mathfrak{L}, \Delta_{t}, \alpha\right)$ co-multiplicative Hom-Lie supercoalgebra, and the compatibility condition (1.8) holds for $\Delta_{t}$ and $[\cdot, \cdot]$.

Precisely we need to prove four things:

- $\alpha^{\otimes 2} \circ \Delta_{t}=\Delta_{t} \circ \alpha$, equality is true because:

$$
\begin{aligned}
\Delta_{t}(\alpha(x)) & =\Delta(\alpha(x))+a d_{\alpha(x)}(t) . \\
\alpha^{\otimes 2}\left(\Delta_{t}(x)\right) & =\alpha^{\otimes 2}(\Delta(x))+\alpha^{\otimes 2}\left(a d_{x}(t)\right)=\Delta(\alpha(x))+\alpha^{\otimes 2}\left(a d_{x}\left(t_{1} \otimes t_{2}\right)\right) .
\end{aligned}
$$

Using $\alpha^{\otimes 2}(t)=t$, we have

$$
\alpha^{\otimes 2}\left(\operatorname{ad}_{x}\left(t_{1} \otimes t_{2}\right)\right)=\left[\alpha(x), t_{1}\right] \otimes \alpha\left(t_{2}\right)+(-1)^{|x|\left|t_{1}\right|} \alpha\left(t_{1}\right) \otimes\left[\alpha(x), t_{2}\right]=a d_{\alpha(x)}(t) .
$$

- $\Delta_{t}$ is skew-supersymmetric because:

$$
\begin{aligned}
\Delta_{t}(x) & =\Delta(x)+a d_{x}(t), \\
\tau\left(\Delta_{t}(x)\right) & =-\Delta(x)+(-1)^{\left|t_{1}\right|\left|t_{2}\right|}\left(\left[x, t_{2}\right] \otimes \alpha\left(t_{1}\right)+(-1)^{|x|\left|t_{2}\right|} \alpha\left(t_{2}\right) \otimes\left[x, t_{1}\right]\right) .
\end{aligned}
$$

Then : $\Delta_{t}(x)+\tau\left(\Delta_{t}(x)\right)=a d_{x}\left(t+(-1)^{\left|t_{1}\right|\left|t_{2}\right|} t_{2} \otimes t_{1}\right)=a d_{x}\left(t+t_{21}\right)=a d_{x}(0)=0$.

- Now, we need to show the compatibility condition (1.8) holds for $\Delta_{t}$ and [., $\left.\cdot\right]$ :

$$
\Delta_{t}([x, y])=a d_{\alpha(x)}\left(\Delta_{t}(y)\right)-(-1)^{|x||y|} a d_{\alpha(y)}\left(\Delta_{t}(x)\right)
$$

which is equivalent to

$$
\Delta_{t}([x, y])=\left|a d_{\alpha(x)}\left(\Delta_{t}(y)\right)\right| .
$$

Since $\Delta_{t}=\Delta+a d(t),(4.3)$ is equivalent to

$$
\Delta([x, y])+a d_{[x, y]}(t)=\left|a d_{\alpha(x)}(\Delta(y))+a d_{\alpha(x)}\left(a d_{y}(t)\right)\right|=\left|a d_{\alpha(x)}(\Delta(y))\right|+\left|a d_{\alpha(x)}\left(a d_{y}(t)\right)\right| .
$$

Moreover, since $\Delta([x, y])=a d_{\alpha(x)}(\Delta(y))-(-1)^{|x||y|} a d_{\alpha(y)}(\Delta(x))=\left|a d_{\alpha(x)}(\Delta(y))\right|$, because $\mathfrak{L}$ is a Hom-Lie superbialgebra, (4.3) is equivalent to, $a d_{[x, y]}(t)=\left|\operatorname{ad}_{\alpha(x)}\left(a d_{y}(t)\right)\right|$, which holds by Lemma 3.11 .

- Finally, we must show the Hom-super-coJacobi identity of $\Delta_{t}$, which states

$$
\circlearrowleft\left(\alpha \otimes \Delta_{t}\right)\left(\Delta_{t}(x)\right)=0
$$

for all $x \in \mathfrak{L}$. Using the definition $\Delta_{t}=\Delta+a d(t)$. We can rewrite (4.4) as

$$
\circlearrowleft(\alpha \otimes \Delta)(\Delta(x))+\circlearrowleft(\alpha \otimes \Delta)\left(a d_{x}(t)\right)+\circlearrowleft(\alpha \otimes a d(t))(\Delta(x))+\circlearrowleft(\alpha \otimes a d(t))\left(a d_{x}(t)\right)=0 .
$$


We already know that $\circlearrowleft(\alpha \otimes \Delta)(\Delta(x))=0$, which is the Hom-super-coJacobi identity of $\Delta$.

Moreover, in (3.8) and (3.9) (in the proof of Theorem 3.12 with $t$ instead of $r$ ), we already showed that

$$
\circlearrowleft(\alpha \otimes a d(t))\left(a d_{x}(t)\right)=\alpha^{\otimes 3}\left(a d_{x}\left([[t, t]]^{\alpha}\right)\right) .
$$

In view of (4.5) and (4.6), the Hom-super-coJacobi identity of $\Delta_{t}$ (4.4) is equivalent to

$$
\alpha^{\otimes 3}\left(a d_{x}\left([[t, t]]^{\alpha}\right)\right)+\circlearrowleft\left((\alpha \otimes a d(t))(\Delta(x))+(\alpha \otimes \Delta)\left(a d_{x}(t)\right)\right)=0 .
$$

Using the assumption (4.1), the condition (4.7) is equivalent to

$$
\circlearrowleft\left((\alpha \otimes a d(t))(\Delta(x))+(\alpha \otimes \Delta)\left(a d_{x}(t)\right)\right)=\alpha^{\otimes 3}\left(\circlearrowleft a d_{x}((\alpha \otimes \Delta)(t))\right) .
$$

We will prove (4.8) in Lemma 4.2 below.

The proof of Theorem 4.1 will be complete once we prove Lemma 4.2 .

Lemma 4.2. The condition 4.8 holds.

Proof. Write $\Delta(x)=\sum x_{1} \otimes x_{2}$ and $t=\sum t_{1} \otimes t_{2} \in \mathfrak{L}^{\otimes 2}$. Then the left-hand side of (4.8) is:

$$
\begin{aligned}
& \left((\alpha \otimes a d(t))(\Delta(x))+(\alpha \otimes \Delta)\left(a d_{x}(t)\right)\right) \\
= & \circlearrowleft\left(\alpha\left(x_{1}\right) \otimes a d_{x_{2}}\left(t_{1} \otimes t_{2}\right)+(\alpha \otimes \Delta)\left(\left[x, t_{1}\right] \otimes \alpha\left(t_{2}\right)+(-1)^{|x|\left|t_{1}\right|} \alpha\left(t_{1}\right) \otimes\left[x, t_{2}\right]\right)\right) \\
= & \circlearrowleft\left(\alpha\left(x_{1}\right) \otimes\left[x_{2}, t_{1}\right] \otimes \alpha\left(t_{2}\right)+(-1)^{\left|x_{2}\right|\left|t_{1}\right|} \alpha\left(x_{1}\right) \otimes \alpha\left(t_{1}\right) \otimes\left[x_{2}, t_{2}\right]\right) \\
+ & \circlearrowleft\left(\alpha\left(\left[x, t_{1}\right]\right) \otimes \Delta\left(\alpha\left(t_{2}\right)\right)+(-1)^{|x|\left|t_{1}\right|} \alpha^{2}\left(t_{1}\right) \otimes \Delta\left(\left[x, t_{2}\right]\right)\right) .
\end{aligned}
$$

Write $\Delta\left(t_{2}\right)=\sum t_{2}^{\prime} \otimes t_{2}^{\prime \prime}$. Recall from (1.8) that: $\Delta\left(\left[x, t_{2}\right]\right)=a d_{\alpha(x)}\left(\Delta\left(t_{2}\right)\right)-(-1)^{|x|\left|t_{2}\right|} a d_{\alpha\left(t_{2}\right)}(\Delta(x))$, because $\mathfrak{L}$ is a Hom-Lie superbialgebra. We can continue the above computation as follows :

$$
\begin{aligned}
& =\circlearrowleft\left(\alpha\left(x_{1}\right) \otimes\left[x_{2}, t_{1}\right] \otimes \alpha\left(t_{2}\right)+(-1)^{\left|x_{2}\right|\left|t_{1}\right|} \alpha\left(x_{1}\right) \otimes \alpha\left(t_{1}\right) \otimes\left[x_{2}, t_{2}\right]+\alpha\left(\left[x, t_{1}\right]\right) \otimes \alpha^{\otimes 2}\left(\Delta\left(t_{2}\right)\right)\right) \\
& +\circlearrowleft\left((-1)^{|x|\left|t_{1}\right|} \alpha^{2}\left(t_{1}\right) \otimes\left[\alpha(x), t_{2}^{\prime}\right] \otimes \alpha\left(t_{2}^{\prime \prime}\right)+(-1)^{|x|\left|t_{1}\right|}(-1)^{|x|\left|t_{2}^{\prime}\right|} \alpha^{2}\left(t_{1}\right) \otimes \alpha\left(t_{2}^{\prime}\right) \otimes\left[\alpha(x), t_{2}^{\prime \prime}\right]\right) \\
& -\circlearrowleft\left((-1)^{|x|\left|t_{1}\right|}(-1)^{|x|\left|t_{2}\right|} \alpha^{2}\left(t_{1}\right) \otimes\left[\alpha\left(t_{2}\right), x_{1}\right] \otimes \alpha\left(x_{2}\right)\right. \\
& \left.-(-1)^{|x|\left|t_{1}\right|}(-1)^{|x|\left|t_{2}\right|}(-1)^{\left|x_{1}\right|\left|t_{2}\right|} \alpha^{2}\left(t_{1}\right) \otimes \alpha\left(x_{1}\right) \otimes\left[\alpha\left(t_{2}\right), x_{2}\right]\right) .
\end{aligned}
$$

It follows the skew-supersymmetry of $\Delta$ applied to $x$ (i.e, $\left.\sum x_{1} \otimes x_{2}=-\sum(-1)^{\left|x_{1}\right|\left|x_{2}\right|} x_{2} \otimes x_{1}\right),|t|=\overline{0}$, $t_{21}=-t$, and $\alpha^{\otimes 2}(t)=t$, We find that

- $\alpha^{2}\left(t_{1}\right) \otimes\left[\alpha\left(t_{2}\right), x_{1}\right] \otimes \alpha\left(x_{2}\right)=(-1)^{\left|x_{1}\right|\left|x_{2}\right|} \alpha\left(x_{2}\right) \otimes \alpha^{2}\left(t_{1}\right) \otimes\left[\alpha\left(t_{2}\right), x_{1}\right]$.

- $(-1)^{\left|x_{1}\right|\left|t_{2}\right|} \alpha^{2}\left(t_{1}\right) \otimes \alpha\left(x_{1}\right) \otimes\left[\alpha\left(t_{2}\right), x_{2}\right]=(-1)^{\left|t_{1}\right|\left|t_{2}\right|}(-1)^{\left|x_{2}\right|\left|t_{1}\right|} \alpha\left(x_{1}\right) \otimes\left[\alpha\left(t_{2}\right), x_{2}\right] \otimes \alpha^{2}\left(t_{1}\right)$. That the first two terms and the last two terms above cancel out. Using the commutation of $\alpha$ with $[\cdot, \cdot]$ and $\Delta$ and $\alpha^{\otimes 2}(t)=t$, the above computation continues as follows:

$$
\begin{aligned}
& =\circlearrowleft\left(\alpha\left(\left[x, t_{1}\right]\right) \otimes \alpha^{\otimes 2}\left(\Delta\left(t_{2}\right)\right)+(-1)^{|x|\left|t_{1}\right|} \alpha^{2}\left(t_{1}\right) \otimes\left[\alpha(x), t_{2}^{\prime}\right] \otimes \alpha\left(t_{2}^{\prime \prime}\right)\right. \\
& \left.+(-1)^{|x|\left(\left|t_{1}\right|+\left|t_{2}^{\prime}\right|\right)} \alpha^{2}\left(t_{1}\right) \otimes \alpha\left(t_{2}^{\prime}\right) \otimes\left[\alpha(x), t_{2}^{\prime \prime}\right]\right) \\
& =\circlearrowleft\left(\alpha\left(\left[x, \alpha\left(t_{1}\right)\right]\right) \otimes \alpha^{\otimes 2}\left(\Delta\left(\alpha\left(t_{2}\right)\right)\right)+(-1)^{|x|\left|t_{1}\right|} \alpha^{3}\left(t_{1}\right) \otimes\left[\alpha(x), \alpha\left(t_{2}^{\prime}\right)\right] \otimes \alpha^{2}\left(t_{2}^{\prime \prime}\right)\right. \\
& \left.+(-1)^{|x|\left(\left|t_{1}\right|+\left|t_{2}^{\prime}\right|\right)} \alpha^{3}\left(t_{1}\right) \otimes \alpha^{2}\left(t_{2}^{\prime}\right) \otimes\left[\alpha(x), \alpha\left(t_{2}^{\prime \prime}\right)\right]\right) \\
& =\alpha^{\otimes 3}\left(\circlearrowleft\left(\left[x, \alpha\left(t_{1}\right)\right] \otimes \Delta\left(\alpha\left(t_{2}\right)\right)+(-1)^{|x|\left|t_{1}\right|} \alpha^{2}\left(t_{1}\right) \otimes\left[x, t_{2}^{\prime}\right] \otimes \alpha\left(t_{2}^{\prime \prime}\right)+(-1)^{|x|\left(\left|t_{1}\right|+\left|t_{2}^{\prime}\right|\right)} \alpha^{2}\left(t_{1}\right) \otimes \alpha\left(t_{2}^{\prime}\right) \otimes\left[x, t_{2}^{\prime \prime}\right]\right)\right) \\
& =\alpha^{\otimes 3}\left(\circlearrowleft a d_{x}\left(\alpha\left(t_{1}\right) \otimes t_{2}^{\prime} \otimes t_{2}^{\prime \prime}\right)\right)=\alpha^{\otimes 3}\left(\circlearrowleft a d_{x}((\alpha \otimes \Delta)(t))\right) .
\end{aligned}
$$

This proves (4.8).

The following result is a special case of the previous theorem.

Corollary 4.3. Let $(\mathfrak{L},[\cdot, \cdot], \Delta, \alpha)$ be a multiplicative Hom-Lie superbialgebra and $t \in \mathfrak{L}^{\otimes 2}$ be an element such that $\alpha^{\otimes 2}(t)=t, \quad t_{21}=-t$, (1.3) and (3.2), (3.2) and (1.3), i.e., $\left.|t|=\overline{0}\right)$ and $[[t, t]]^{\alpha}+\circlearrowleft(\alpha \otimes \Delta)(t)=0$ for all $x \in \mathfrak{L}$. Then $\mathfrak{L}_{t}=\left(\mathfrak{L},[\cdot, \cdot], \Delta_{t}=\Delta+\operatorname{ad}(t), \alpha\right)$ is multiplicative Hom-Lie superbialgebra. 


\section{References}

[1] Ammar F. and Makhlouf A., Hom-Lie superalgebras and Hom-Lie admissible superalgebras, Journal of Algebra, Vol. 324 (7), (2010) 1513-1528.

[2] Ammar F., Makhlouf A. and Saadaoui N., Cohomology of Hom-Lie superalgebras and q-deformed Witt superalgebra, Czechoslovak Mathematical Journal, Vol. 63, No. 3, (2013) 721-761.

[3] Andruskiewitsch N., Lie Superbialgebras and Poisson-Lie Supergroups, Abh. Math. Sem. Univ. Hamburg 63 (1993), 147-163.

[4] Benayadi S. and Makhlouf A., Hom-Lie algebras with symmetric invariant nonDegenerate bilinear forms, Journal of Geometry and Physics 76 (2014) 38-60.

[5] Cai L. and Sheng Y, Purely Hom-Lie bialgebras, arXiv:1605.00722 (2016) to appear in Sci. China Math.

[6] Drinfel'd V.G., Constant quasiclassical solution of the Yang-Baxter quantum equation, Sov. Math. Dokl., 28 (1983), 667-671.

[7] Drinfel'd V.G., Hamiltonian structures on Lie groups, Lie bialgebras and the geometric meaning of classical Yang-Baxter equations, Sov., Dokl. Akad.Nauk SSSR 268 (1983), no. 2, 285-287. English translation: Soviet Math. Dokl. 27 (1983), no.1, 68-71.

[8] Drinfel'd V. G., Quantum Groups, in: Proc. ICM (Berkeley, 1986), p.798-820, AMS, Providence, RI, 1987.

[9] Drinfel'd V.G., Quasi-Hopf Algebras and Knizhnik-Zamolodchikov Equation, in: A.U. Klimyk, et al ed., Problems of Modern Quantum Field Theory, p.1-13, Springer, Berlin, 1989.

[10] Drinfel'd V.G., Quasi-Hopf algebras, Leningrad Math. J., 1(6) (1990), 1419-1457.

[11] Drinfel'd V.G., Structure of the quasitriangular quasi-Hopf algebras, Functional Anal. Appl., 26:1(1992), 63-65.

[12] Hartwig J., Larsson D. and Silvestrov S., Deformations of Lie algebras using $\sigma$-derivations, J. Algebra 295 (2006), 314-361.

[13] Hengyun Y. and Yucai S., Lie superbialgebra structures on generalized super-Virasoro algebras. Acta Mathematica Scientia 2010, 30B(1):225-239.

[14] Larsson D. and Silvestrov S., Quasi-hom-Lie algebras, central extensions and 2-cocycle-like identities, J. Algebra, 288 (2005), 321-344.

[15] Larsson D. and Silvestrov S., Quasi-Lie algebras, Contemp. Math., 391 (2005), 241-248.

[16] Majid S., Foundation of Quantum Group Theory, Cambridge University Press, Cambridge, UK, 1995.

[17] Makhlouf A., Paradigm of nonassociative Hom-algebras and Hom-superalgebras, Proceedings of Jordan Structures in Algebra and Analysis Meeting, Editorial Circulo Rojo, Almeria : 143-177, 2010.

[18] Makhlouf A. and Silvestrov S., Hom-algebra structures, J. Gen. Lie Theory Appl. 2 (2008), 51-64.

[19] Makhlouf A. and Silvestrov S., Hom-algebras and Hom-coalgebras, J. Alg. Appl., 9(4) (2010), 1-37.

[20] Makhlouf A. and Silvestrov S., Notes on 1-parameter formal deformations of Hom-associative and HomLie algebras, Forum Math., 22 (2010), 715-739.

[21] Makhlouf A. and Yau D., Rota-Baxter Hom-Lie-admissible algebras, Comm. Algebra, 42(3) (2014), 1231-1257.

[22] Sheng Y., Representation of Hom-Lie algebras, Algebr. Represent. Theory, 15 (2012), 1081-1098.

[23] Sheng Y, Bai C., A new approach to Hom-Lie bialgebras, Journal of Algebra 399 (2014) 232-250. 
[24] Walter M., Lie Coalgebras, Advances in Mathematics 38, 1-54 (1980).

[25] Yau D., Enveloping algebras of Hom-Lie algebras, J. Gen. Lie Theory Appl., 2(2) (2008), 95-108.

[26] Yau D., Hom-algebras and homology, J. Lie Theory, 19(2) (2009), 409-421.

[27] Yau D., The classial Hom-Yang-Baxter equation and Hom-Lie bialgebras, International Electronic Journal of Algebra Volume 17 (2015) 11-45.

\section{Addresses.}

M. Fadous, University of Sfax, Faculty of Sciences Sfax, BP 1171, 3038 Sfax, Tunisia.

S. Mabrouk, Université de Gafsa, Faculté des Sciences, Gafsa Tunisia

A. Makhlouf, University of Haute Alsace, 4 rue des frères Lumière, 68093 Mulhouse France.

Emails. mohamedfadous201001@gmail.com

mabrouksami00@yahoo.fr

Abdenacer.Makhlouf@uha.fr 